\title{
THE FAST AND FURIOUS DECAY OF THE PECULIAR TYPE Ic SUPERNOVA 2005ek
}

\author{
M. R. Drout ${ }^{1}$, A. M. Soderberg ${ }^{1}$, P. A. Mazzali ${ }^{2,3,4}$, J. T. Parrent ${ }^{5,6}$, R. Margutti $^{1}$, D. Milisavljevic ${ }^{1}$, N. E. Sanders ${ }^{1}$, \\ R. Chornock ${ }^{1}$, R. J. Foley ${ }^{1}$, R. P. Kirshner ${ }^{1}$, A. V. Filippenko ${ }^{7}$, W. Li ${ }^{7}{ }^{14}$, P. J. Brown ${ }^{8}$, S. B. Cenko ${ }^{7}$, S. Chakraborti ${ }^{1}$,

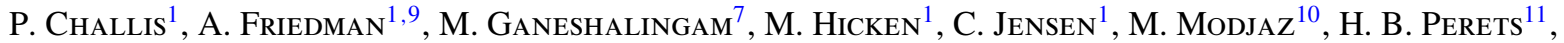 \\ J. M. SilvermaN ${ }^{12,15}$, AND D. S. WONG ${ }^{13}$ \\ ${ }^{1}$ Harvard-Smithsonian Center for Astrophysics, 60 Garden Street, Cambridge, MA 02138, USA; mdrout@ cfa.harvard.edu \\ ${ }^{2}$ Astrophysics Research Institute, Liverpool John Moores University, CH41 1LD Liverpool, UK \\ ${ }^{3}$ INAF-Osservatorio Astronomico di Padova, Vicolo dell'Osservatorio 5, I-35122 Padova, Italy \\ ${ }^{4}$ Max-Planck-Institut for Astrophysik, Karl-Schwarzschildstr. 1, D-85748 Garching, Germany \\ ${ }^{5}$ Department of Physics and Astronomy, Dartmouth College, 6127 Wilder Laboratory, Hanover, NH 03755, USA \\ ${ }^{6}$ Las Cumbres Observatory Global Telescope Network, Goleta, CA 93117, USA \\ ${ }^{7}$ Department of Astronomy, University of California, Berkeley, CA 94720-3411, USA \\ ${ }^{8}$ Department of Physics and Astronomy, Texas A\&M University, College Station, TX 77843-4242, USA \\ ${ }^{9}$ Center for Theoretical Physics, Massachusetts Institute of Technology, 77 Massachusetts Avenue, 6-304, Cambridge, MA 02139, USA \\ ${ }^{10}$ Center for Cosmology and Particle Physics, Department of Physics, New York University, 4 Washington Place, New York, NY 10003, USA \\ ${ }^{11}$ Physics Department, Technion-Israel Institute of Technology, 32000 Haifa, Israel \\ ${ }^{12}$ Department of Astronomy, University of Texas at Austin, Austin, TX 78712, USA \\ ${ }^{13}$ Physics Department, University of Alberta, 4-183 CCIS, Edmonton, AB T6G 2E1, Canada \\ Received 2013 June 10; accepted 2013 July 10; published 2013 August 16
}

\begin{abstract}
We present extensive multi-wavelength observations of the extremely rapidly declining Type Ic supernova (SN Ic), SN 2005ek. Reaching a peak magnitude of $M_{R}=-17.3$ and decaying by $\sim 3$ mag in the first 15 days post-maximum, SN 2005ek is among the fastest Type I supernovae observed to date. The spectra of SN $2005 \mathrm{ek}$ closely resemble those of normal SN Ic, but with an accelerated evolution. There is evidence for the onset of nebular features at only nine days post-maximum. Spectroscopic modeling reveals an ejecta mass of $\sim 0.3 M_{\odot}$ that is dominated by oxygen $(\sim 80 \%)$, while the pseudo-bolometric light curve is consistent with an explosion powered by $\sim 0.03 M_{\odot}$ of radioactive ${ }^{56} \mathrm{Ni}$. Although previous rapidly evolving events (e.g., SN 1885A, SN 1939B, SN 2002bj, SN 2010X) were hypothesized to be produced by the detonation of a helium shell on a white dwarf, oxygen-dominated ejecta are difficult to reconcile with this proposed mechanism. We find that the properties of SN 2005ek are consistent with either the edge-lit double detonation of a low-mass white dwarf or the iron-core collapse of a massive star, stripped by binary interaction. However, if we assume that the strong spectroscopic similarity of SN 2005ek to other SNe Ic is an indication of a similar progenitor channel, then a white-dwarf progenitor becomes very improbable. SN 2005ek may be one of the lowest mass stripped-envelope core-collapse explosions ever observed. We find that the rate of such rapidly declining Type I events is at least $1 \%-3 \%$ of the normal SN Ia rate.
\end{abstract}

Key words: supernovae: general - supernovae: individual (SN2005ek)

Online-only material: color figures

\section{INTRODUCTION}

The advent of dedicated supernova ( $\mathrm{SN}$ ) searches has dramatically increased the rate at which unusual transients are discovered. In particular, high-cadence surveys have uncovered a diverse set of rapidly evolving events which reach SN luminosities (absolute magnitude between -20 and -15) but have observed properties that challenge the parameter space easily explained by traditional SN models (e.g., the collapse of the core of a massive star, or the thermonuclear disruption of a white dwarf (WD)).

The plethora of objects that have been referred to as "rapidly evolving" include both Type I (hydrogen poor) and Type II (hydrogen rich) events (see Filippenko 1997 for a review of traditional SN classifications). Although the main physical process leading to optical emission varies among $\mathrm{SNe}$, in all cases the characteristic timescale offers insight into the amount of participating material. Rapid evolution typically implies

\footnotetext{
${ }^{14}$ Deceased 2011 December 12.

${ }^{15}$ NSF Astronomy and Astrophysics Postdoctoral Fellow.
}

lower masses. For SNe powered by hydrogen recombination (e.g., Types IIP, IIL) and radioactive decay (e.g., Types Ia, Ib, Ic), rapid timescales indicate a low hydrogen envelope mass and a short photon diffusion timescale, respectively. For SNe powered by interaction with external gas (e.g., Type IIn), a rapid decline implies a steep decrease in circumstellar medium (CSM) density, and a short overall timescale implies a small radius over which this material is located.

Among the Type I events labeled as rapidly evolving are the SN 1991bg-like SN Ia (Filippenko et al. 1992; Leibundgut et al. 1993), the "calcium-rich" transients for which SN 2005E is the prototype (Perets et al. 2010; Kasliwal et al. 2012; Valenti et al. 2013), and some members of the recently defined Type Iax SNe (Foley et al. 2013). They earn the title "rapidly evolving" because they decay by 1-2 mag in the first 15 days postmaximum. Many of these objects possess peak luminosities lower than those of normal SN I ( $\gtrsim-15 \mathrm{mag}$ ) and are thought to be powered by radioactive decay. Although their host galaxies are diverse, members of the first two classes above have exploded in elliptical galaxies. In addition, several luminous $(M \lesssim-19 \mathrm{mag})$ transients have been observed that decay on 
similar timescales, but show narrow hydrogen and/or helium emission lines in their spectra, indicating that they are at least partially powered by interaction with a dense CSM. These include the Type IIn SN PTF09uj (Ofek et al. 2010) and the Type Ibn SN 1999cq (Matheson et al. 2000).

However, the record for the most rapidly declining SN observed thus far does not belong to any of these objects. SN 2002bj (Poznanski et al. 2010) and SN 2010X (Kasliwal et al. 2010) easily outstrip them, declining by $\gtrsim 3$ mag in the first 15 days post-maximum. The ejecta masses inferred for these two events are very small $\left(\lesssim 0.3 M_{\odot}\right)$, but their peak luminosities are within the typical range for SN Ib/Ic $(-19 \lesssim M \lesssim-17$; Drout et al. 2011). These two facts, coupled with the lack of hydrogen in their spectra (SN 2002bj is an SN Ib, SN 2010X an SN Ic), have led several authors to hypothesize that they were produced by the detonation of a helium shell on a WD (a ".Ia" SN; Woosley et al. 1986; Chevalier \& Plait 1988; Bildsten et al. 2007; Shen et al. 2010; Waldman et al. 2011; Sim et al. 2012).

Two potential other members of this class include SN 1885A and SN 1939B (see, e.g., Perets et al. 2011; Chevalier \& Plait 1988; de Vaucouleurs \& Corwin 1985; Leibundgut et al. 1991). While both SN 2002bj and SN 2010X were found in starforming galaxies, SN 1939B exploded in an elliptical, a fact suggestive of an old progenitor system. However, while the postmaximum decline rates of these objects are similar, even the well-studied events show differences in their other properties. SN 2002bj was $\sim 1.5$ mag brighter, significantly bluer, and exhibited lower expansion velocities than SN 2010X. It has not yet been established whether all (or any) of these extremely rapidly declining objects belong to the same class of events.

Here we present the discovery and panchromatic followup observations of SN 2005ek, another very rapidly declining and hydrogen-free event that closely resembles SN 2010X. In Section 2 we present our extensive multi-wavelength observations, while in Sections 3-6 we respectively describe the photometric and spectroscopic properties, explosion parameters, and host-galaxy environment of SN 2005ek. Section 7 examines the rates of such transients. Finally, in Section 8, we discuss progenitor channels that could lead to such a rapidly evolving explosion.

\section{OBSERVATIONS}

\subsection{Discovery}

SN 2005ek was discovered by the Lick Observatory Supernova Search (LOSS) using the Katzman Automatic Imaging Telescope (KAIT; Filippenko et al. 2001) on 2005 September 24.53 (UT dates are used throughout this paper) with an unfiltered (clear) $m \approx 17.5 \mathrm{mag}$. The object was not detected in previous KAIT images on September 18.51 to a limit of $m \approx 19$ mag, while subsequent imaging on September 25.37 revealed that the transient had brightened to $m \approx 17.3 \mathrm{mag}$ (Khandrika \& Li 2005). SN 2005ek is located in the outskirts of its host galaxy, UGC 2526, with distance $D=66.6 \pm 4.7 \mathrm{Mpc}^{16}$ and morphology $\mathrm{Sb}$.

Wong et al. (2005) obtained a spectrum of SN 2005ek on September 26 with the Shane $3 \mathrm{~m}$ reflector (plus Kast spectrograph) at Lick Observatory and reported that SN 2005ek was a "young SN, probably of Type Ic." After this spectroscopic identification, we promptly initiated a panchromatic follow-up

\footnotetext{
16 We adopt the NED distance after correction for Virgo, Great Attractor, and Shapley Supercluster Infall and assuming $H_{0}=73 \mathrm{~km} \mathrm{~s}^{-1} \mathrm{Mpc}^{-1}$ (Mould et al. 2000).
}

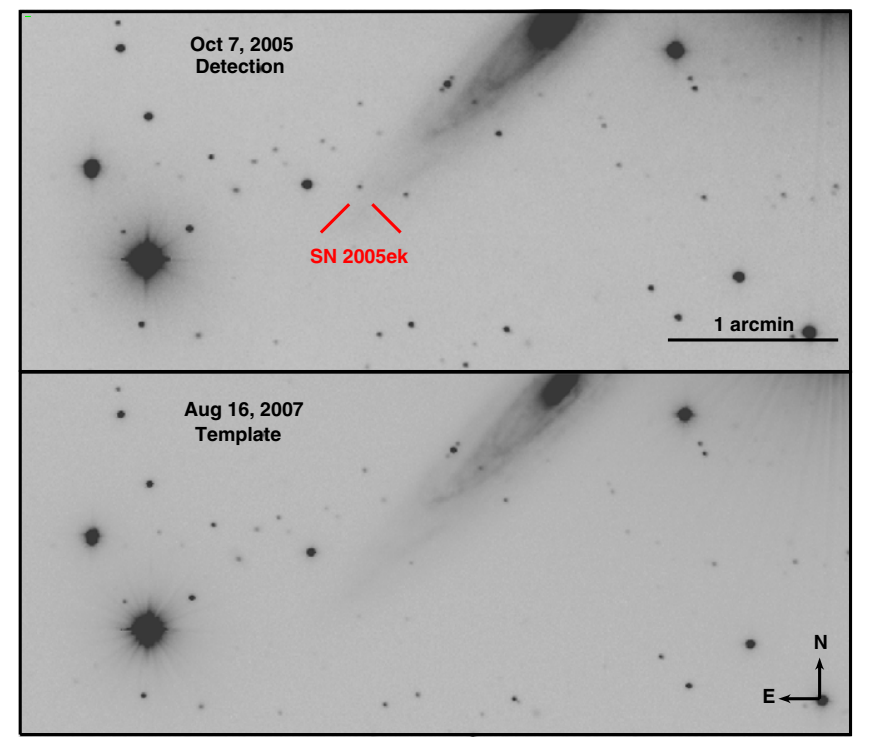

Figure 1. Top: $R$-band Palomar 60 inch (P60) image of SN 2005ek, on the outskirts of UGC 2526. The SN location is marked by red crosshairs. Bottom: P60 template image of the region around SN 2005ek, taken on 2007 August 26. (A color version of this figure is available in the online journal.)

program spanning the radio, infrared (IR), optical, ultraviolet (UV), and X-ray bands.

\subsection{Palomar 60 Inch Imaging}

We obtained nightly multi-band images of SN 2005ek with the robotic Palomar 60 inch telescope (P60; Cenko et al. 2006) beginning on September 26.3 and spanning through October 15.2. Each epoch consisted of 4-10 $120 \mathrm{~s}$ frames in filters $B, V, R$, and $I$. All P60 images were reduced with IRAF ${ }^{17}$ using a custom real-time reduction pipeline (Cenko et al. 2006). Nightly images were combined using standard IRAF tools. Images of the transient and host galaxy constructed from P60 data are shown in Figure 1.

For the P60 $V$ and $R$ bands we obtained template images of the region surrounding SN 2005ek on 2007 August 16 (bottom panel of Figure 1), after the SN had faded from view. We subtracted the host-galaxy emission present in the template image using a common point-spread function (PSF) method and then performed aperture photometry on the resulting difference images. For the $B$ and $I$ bands, no suitable template images were obtained, and we therefore performed PSF photometry on our stacked images directly. A comparison of these two methods with our $V$-and $R$-band data revealed that the resulting photometry was consistent within the measured uncertainties.

In all cases, we measured the relative magnitude of the $\mathrm{SN}$ with respect to five field stars within the full $13^{\prime} \times 13^{\prime} \mathrm{P} 60$ field of view. Absolute calibration was performed based on Sloan Digital Sky Survey (SDSS) photometry of the field stars (Ahn et al. 2012), converted to the BVRI system using the relations from Smith et al. (2002). Our resulting P60 photometry is listed in Table 1 and shown as filled circles in the main panel of Figure 2. These data reveal that the light curve reaches maximum at $m_{R} \approx 17.4$ mag only $\sim 9$ days after the KAIT nondetection and subsequently decays very rapidly in all bands.

\footnotetext{
17 IRAF is distributed by the National Optical Astronomy Observatory, which is operated by the Association for Research in Astronomy, Inc. under cooperative agreement with the National Science Foundation.
} 


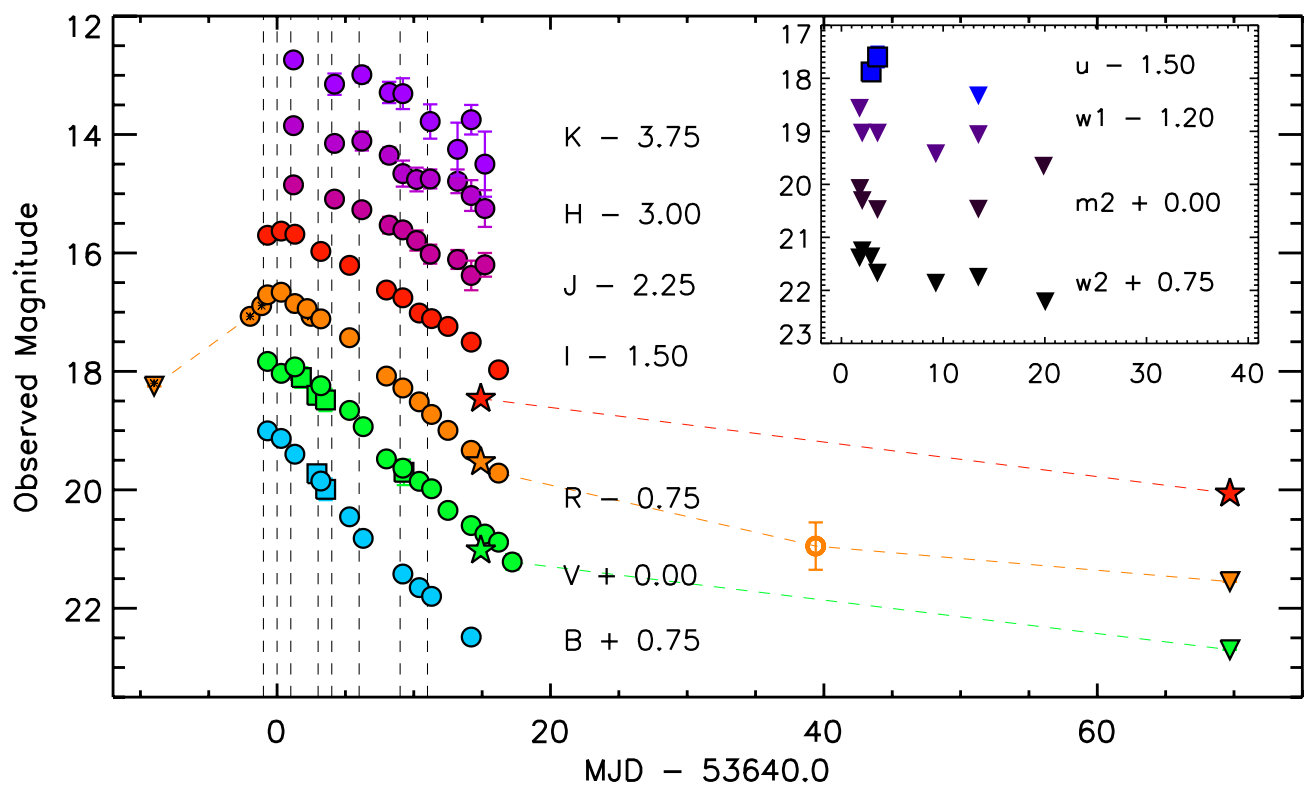

Figure 2. Photometry of SN 2005ek. P60 BVRI- and PAIRITEL $J H$ K -band data (circles) are respectively shown as cyan, green, orange, red, and three shades of violet. Lick Observatory discovery observations and the pre-discovery nondetection are shown as orange circles and a triangle with an asterisk inside. P200 gri-band data are shown as green, orange, and red stars; triangles denote upper limits. A late-time FLWO $r$-band detection is shown as an open orange circle. UVOT $b$ - and $v$-band detections are shown as blue and green squares in the main panel. UVOT $u-, w 1-, m 2-$, and $w 2$ detections (squares) and upper limits (triangles) are shown in the inset. Vertical lines in the main panel indicate epochs on which spectroscopy was obtained.

(A color version of this figure is available in the online journal.)

Table 1

P60 Photometry

\begin{tabular}{lccccc}
\hline \hline UT Date & MJD & $\begin{array}{l}B \text { (err) } \\
(\mathrm{mag})\end{array}$ & $\begin{array}{l}V(\mathrm{err}) \\
(\mathrm{mag})\end{array}$ & $\begin{array}{r}R \text { (err) } \\
(\mathrm{mag})\end{array}$ & $\begin{array}{l}I \text { (err) } \\
(\mathrm{mag})\end{array}$ \\
\hline
\end{tabular}

2005 Sep $26 \quad 53639.3 \quad 18.25(0.02) \quad 17.83(0.02) \quad 17.46(0.01) \quad 17.20(0.02)$ 2005 Sep $27 \quad 53640.3 \quad 18.38(0.03) \quad 18.03(0.03) \quad 17.41(0.02) \quad 17.13(0.04)$ 2005 Sep $2853641.3 \quad 18.65(0.02) \quad 17.92(0.01) \quad 17.60(0.01) \quad 17.18(0.02)$

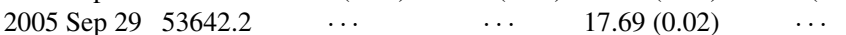

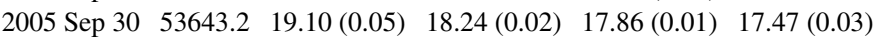
2005 Oct $2 \quad 53645.3 \quad 19.71(0.07) \quad 18.66(0.02) \quad 18.18(0.01) \quad 17.71(0.02)$ $\begin{array}{llllll}2005 \text { Oct } 3 & 53646.5 & 20.07(0.07) & 18.93(0.02) & \ldots & \ldots\end{array}$ $\begin{array}{llllll}2005 \text { Oct } 5 & 53648.0 & \ldots & 19.48(0.06) & 18.83(0.03) & 18.13(0.06)\end{array}$

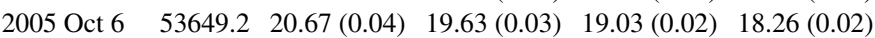
$\begin{array}{lllllll}2005 \text { Oct } 7 & 53650.4 & 20.90(0.04) & 19.86(0.03) & 19.26(0.02) & 18.51(0.02)\end{array}$ $\begin{array}{llllll}2005 \text { Oct } 8 & 53651.3 & 21.05(0.04) & 19.98(0.04) & 19.48(0.02) & 18.61(0.02)\end{array}$ $\begin{array}{llllll}2005 \text { Oct } 9 & 53652.5 & \ldots & 20.35(0.05) & 19.75(0.04) & 18.74(0.03)\end{array}$ 2005 Oct $11 \quad 53654.221 .74(0.12) \quad 20.60(0.08) \quad 20.08(0.05) \quad 19.01(0.05)$ 2005 Oct $1253655.2 \quad \ldots \quad 20.74(0.10) \quad \ldots \quad \ldots$ 2005 Oct $13 \quad 52656.2 \quad \ldots \quad 20.88(0.08) 20.47(0.08) \quad 19.47(0.06)$ 2005 Oct 1453657.2

\subsection{Palomar 200 Inch Imaging}

On 2005 October 11 and December 5 we imaged SN 2005ek with the Large Format Camera mounted on the Palomar 200 inch $(5 \mathrm{~m})$ telescope in the $g^{\prime}, r^{\prime}$, and $i^{\prime}$ bands (120 s exposures). Image processing and PSF photometry were performed using standard packages within IRAF. We performed our absolute calibration using SDSS photometry of field stars, in the same manner as described above. Our resulting photometry is listed in Table 2 and supplements the P60 data in the main panel of Figure 2. On our final epoch, the transient was only detected in the $i^{\prime}$ band at $\sim 21.5 \mathrm{mag}$.

\subsection{Lick Observatory Imaging}

The LOSS unfiltered images of SN 2005ek were reanalyzed for this work in the manner described by Li et al. (2003). These
Table 2

KAIT/P200 Photometry

\begin{tabular}{lcccc}
\hline \hline UT Date & MJD & Telescope & Filter & Mag (err) \\
\hline 2005 Sep 18 & 53631.5 & KAIT & clear & $\lesssim 19$ \\
2005 Sep 24 & 53637.5 & KAIT & clear & $17.82(0.08)$ \\
2005 Sep 25 & 53638.4 & KAIT & clear & $17.64(0.09)$ \\
2005 Sep 29 & 53642.5 & KAIT & clear & $17.82(0.07)$ \\
2005 Oct 11 & 53654.9 & P200 & $g^{\prime}$ & $21.02(0.01)$ \\
2005 Oct 11 & 53654.9 & P200 & $r^{\prime}$ & $20.28(0.01)$ \\
2005 Oct 11 & 53654.9 & P200 & $i^{\prime}$ & $19.96(0.01)$ \\
2005 Dec 5 & 53709.5 & P200 & $g^{\prime}$ & $<22.7$ \\
2005 Dec 5 & 53709.5 & P200 & $r^{\prime}$ & $<22.3$ \\
2005 Dec 5 & 53709.5 & P200 & $i^{\prime}$ & $21.56(0.03)$ \\
\hline
\end{tabular}

include the discovery images (September 24.5 and 25.4) as well as an additional detection on September 29.5 (orange circles with asterisks in Figure 2; also see Table 2). These detections indicate that SN 2005ek was discovered on the rise, while our P60 observations, beginning on September 26.3, show a continued rise for at most one day before a very rapid decline.

\subsection{FLWO Imaging}

We supplement our photometry with the $J H K$ data presented by Modjaz (2007; violet circles in Figure 2). These data were obtained on the PAIRITEL telescope at the Fred Lawrence Whipple Observatory (FLWO) and are well sampled over the same time period as the P60 observations described above. In addition, Modjaz (2007) obtained an $r^{\prime}$-band detection with the FLWO $1.2 \mathrm{~m}$ telescope on 2005 November 5 (open orange circle; Figure 2).

\subsection{Swift/UVOT Imaging}

Swift/UVOT (Roming et al. 2005) observations of SN 2005ek were triggered beginning on 2005 September 29. Seven epochs were obtained in the $u v w 2, u v m 2, u v w 1, u, b$, and/or $v$ filters 
Table 3

UVOT Photometry

\begin{tabular}{lccccccc}
\hline \hline UT Date & MJD & $\begin{array}{c}u v w 2(\mathrm{err}) \\
(\mathrm{mag})\end{array}$ & $\begin{array}{c}u v m 2(\mathrm{err}) \\
(\mathrm{mag})\end{array}$ & $\begin{array}{c}u v w 1(\mathrm{err}) \\
(\mathrm{mag})\end{array}$ & $\begin{array}{c}u(\mathrm{err}) \\
(\mathrm{mag})\end{array}$ & $\begin{array}{c}b(\mathrm{err}) \\
(\mathrm{mag})\end{array}$ & $\begin{array}{c}v(\mathrm{err}) \\
(\mathrm{mag})\end{array}$ \\
\hline 2005 Sep 28 & 53641.8 & $<20.6$ & $<20.1$ & $<19.8$ & $\ldots$ & $\ldots$ & $18.10(0.17)$ \\
2005 Sep 29 & 53642.0 & $<20.5$ & $<20.3$ & $<20.2$ & $\ldots$ & $\ldots$ & $\ldots$ \\
2005 Sep 29 & 53642.9 & $<20.6$ & $\ldots$ & $\ldots$ & $19.28(0.15)$ & $18.97(0.10)$ & $18.40(0.11)$ \\
2005 Sep 30 & 53643.5 & $<20.9$ & $<20.5$ & $<20.2$ & $19.10(0.19)$ & $19.24(0.18)$ & $18.48(0.19)$ \\
2005 Oct 6 & 53649.3 & $<21.1$ & & $<20.6$ & $\ldots$ & $\ldots$ & $19.70(0.22)$ \\
2005 Oct 10 & 53653.5 & $<21.0$ & $<20.5$ & $<20.3$ & $<19.8$ & $<19.8$ & $<19.6$ \\
2005 Oct 16 & 53659.9 & $<21.5$ & $<19.7$ & $\ldots$ & $\ldots$ & $\ldots$ & $<19.9$ \\
\hline
\end{tabular}

Table 4

Optical Spectroscopy

\begin{tabular}{lccccc}
\hline \hline UT Date & MJD & $\begin{array}{c}\text { Phase }^{\mathrm{a}} \\
\text { (days) }\end{array}$ & Target & Telescope & Instrument \\
\hline 2005 Sep 26 & 53639 & -1 & SN 2005ek & Shane 3 m & Kast \\
2005 Sep 27 & 53640 & 0 & SN 2005ek & Tillinghast 60 inch & FAST \\
2005 Sep 28 & 53641 & +1 & SN 2005ek & Tillinghast 60 inch & FAST \\
2005 Sep 30 & 53643 & +3 & SN 2005ek & Tillinghast 60 inch & FAST \\
2005 Oct 1 & 53644 & +4 & SN 2005ek & Tillinghast 60 inch & FAST \\
2005 Oct 3 & 53646 & +6 & SN 2005ek & Tillinghast 60 inch & FAST \\
2005 Oct 6 & 53649 & +9 & SN 2005ek & Keck-II & DEIMOS \\
2005 Oct 8 & 53651 & +11 & SN 2005ek & HET & LRS \\
2011 Feb 22 & 55614 & $\cdots$ & UGC 2526 & MMT & Blue Channel \\
2011 Feb 23 & 55615 & $\cdots$ & UGC 2526 & MMT & Blue Channel \\
\hline
\end{tabular}

Note. ${ }^{a}$ With respect to $R$-band maximum light.

over a time period of 20 days. The data were analyzed following the prescriptions of Brown et al. (2009) and photometry is based on the UVOT photometric system of Poole et al. (2008) with the sensitivity corrections and revised UV zeropoints of Breeveld et al. (2011). All data are listed in Table 3. The uvw2-, uvm2-, $u v w 1-$, and $u$-band data are shown in the inset of Figure 2, while the $b$ - and $v$-band data are plotted as squares in the main panel. The flux from SN 2005ek appears to fall off in the blue, with only upper limits obtained in the UV bands. The spectral energy distribution (SED) of SN 2005ek will be examined in more detail in Section 3.5.

\subsection{Optical Spectroscopy}

Between 2005 September 17 and October 8, we obtained eight low-resolution spectra of SN 2005ek from the FAST spectrograph (Fabricant et al. 1998) on the FLWO 60 inch Tillinghast telescope, the Kast double spectrograph (Miller \& Stone 1993) on the $3 \mathrm{~m}$ Shane reflector at Lick Observatory, the DEIMOS spectrograph (Faber et al. 2003) mounted on the Keck-II $10 \mathrm{~m}$ telescope, and the $9.2 \mathrm{~m}$ Hobby-Eberly Telescope (HET) at McDonald Observatory. Technical details for all of our spectroscopic observations are summarized in Table 4, and the epochs on which they were obtained are marked by dashed vertical lines in Figure 2.

The spectra were reduced in the manner described by Matheson et al. (2008), Blondin et al. (2012), and Silverman et al. (2012). Standard IRAF routines were used to subtract the overscan region and flatfield the two-dimensional CCD frames using a combined and normalized flatfield image. Onedimensional spectra were extracted and wavelength calibrated using comparison lamps obtained immediately following each exposure. The FAST, Kast, and DEIMOS spectra were flux calibrated utilizing a set of custom IDL routines which fit spectrophotometric standards to the data. In addition, these routines apply a small shift to the wavelength calibration after crosscorrelating night-sky lines with a template night-sky spectrum, apply a heliocentric correction, and use the spectrophotometric standards to remove telluric absorption features from the SN spectra (see, e.g., Matheson et al. 2000). The HET spectrum was flux calibrated in IRAF and no telluric correction was made.

All eight spectra are displayed in the top panel of Figure 3, where the FAST and Kast spectra have been smoothed with windows of $11 \AA$ and $7 \AA$, respectively. A thorough analysis of the spectroscopic features and evolution of SN 2005ek will be performed in Section 4. The spectra closely resemble those of the rapidly declining SN 2010X (bottom left panel of Figure 3).

We obtained two spectra of the host galaxy, UGC 2526, with the Blue Channel spectrograph (Schmidt et al. 1989) on the 6.5 m MMT on 2011 February 22 and 23. The first observation was positioned on the explosion site, while the second was on the galactic nucleus and aligned along the major axis of the galaxy. No strong nebular emission lines were detected in the explosion-site spectrum. Reduction, extraction, flux calibration, and telluric correction were performed in the manner described above, and the final spectrum centered on the galaxy nucleus is shown in the bottom right panel of Figure 3. Weak, narrow $\mathrm{H} \alpha$ and $[\mathrm{N}$ II] emission lines are evident, along with a red continuum.

\subsection{Radio Observations}

We observed SN 2005ek with the Very Large Array (VLA) on 2005 September 29 under our target-of-opportunity program to study the nonthermal properties of local Type Ib/Ic SNe. ${ }^{18} \mathrm{At}$ $8.46 \mathrm{GHz}$ we did not detect a coincident radio source, and we place an upper limit of $F_{v} \lesssim 128 \mu \mathrm{Jy}(2 \sigma)$ on the flux density. At a distance of $\sim 67 \mathrm{Mpc}$, this corresponds to a radio luminosity

\footnotetext{
18 VLA Intensive Survey of Naked Supernovae; VISiONS (Soderberg 2007).
} 

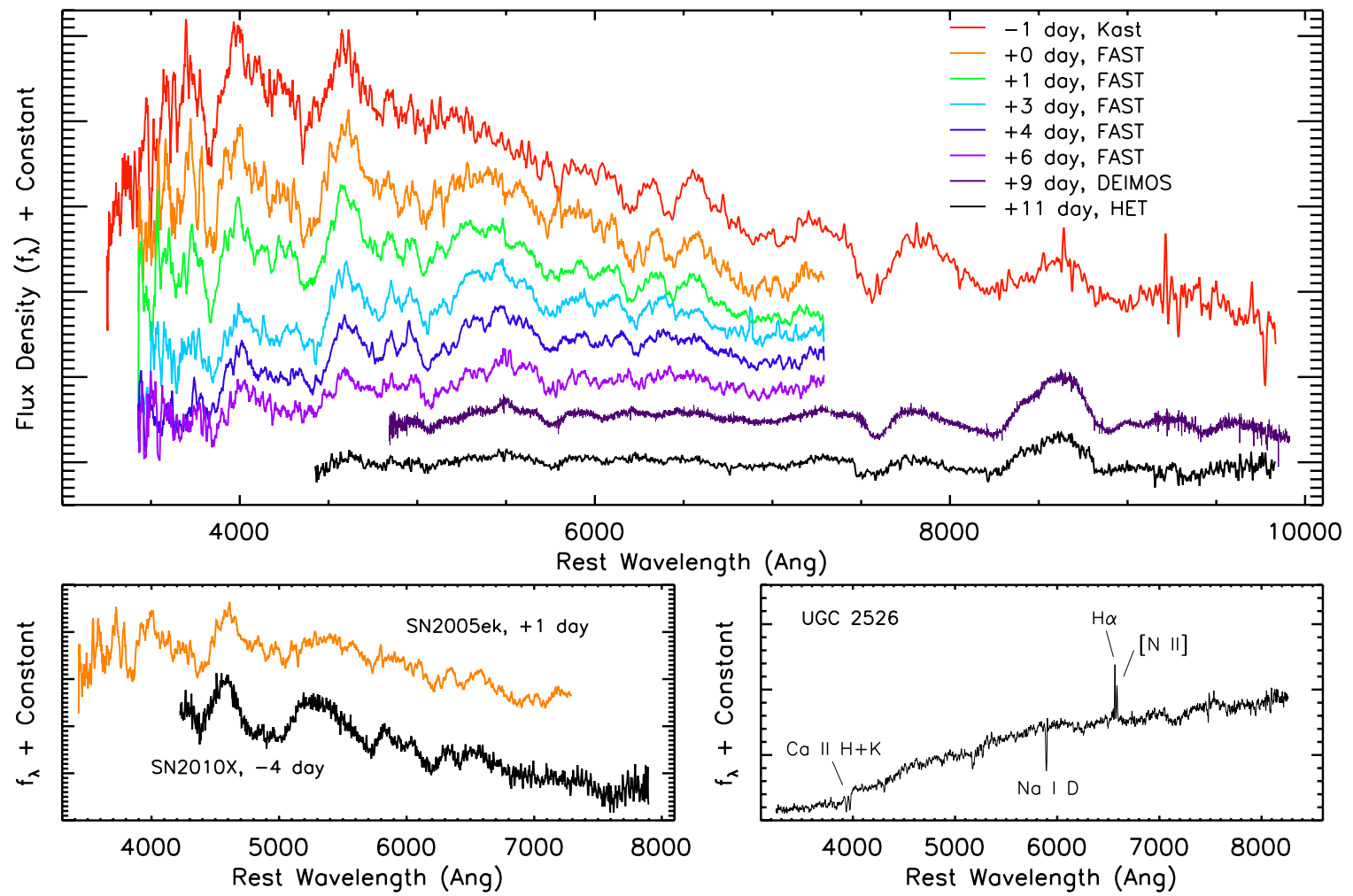

Figure 3. Top: optical spectra of SN 2005ek (see text for details). FAST and Kast spectra have been smoothed with windows of $11 \AA ̊$ and $7 \AA$, respectively. Lower left: comparison of the +1 day spectrum of SN 2005ek with the -4 day spectrum of SN 2010X (Kasliwal et al. 2010). Lower right: spectrum of the host galaxy, UGC 2526. Major features are labeled.

(A color version of this figure is available in the online journal.)

of $L_{v} \lesssim 7 \times 10^{26} \mathrm{erg} \mathrm{s}^{-1} \mathrm{~Hz}^{-1}$. This value is a factor of $\sim 7$ above the peak radio luminosity observed for the subluminous radio SN SN 2007gr (Soderberg et al. 2010).

\subsection{X-Ray Observations}

SN 2005ek was also observed with the X-Ray Telescope (XRT; Burrows et al. 2005) on board Swift (Gehrels et al. 2004) beginning on 2005 September 28 . The data were analyzed using the latest version of the HEASOFT package available at the time of writing (ver. 6.13) and corresponding calibration files. Standard filtering and screening criteria were applied. All XRT data were coadded, resulting in a final $13 \mathrm{ks}$ map spanning 19 days (median time of arrival $=7.44$ days). No X-ray source is detected coincident with SN 2005ek with a $3 \sigma$ upper limit of $F_{x}<1.3 \times 10^{13} \mathrm{erg} \mathrm{s}^{-1} \mathrm{~cm}^{-2}$ (unabsorbed, 0.3-10 keV energy band). The Galactic neutral hydrogen column density in the direction of the SN is $1.0 \times 10^{21} \mathrm{~cm}^{-2}$ (Kalberla et al. 2005). At $D \approx 67 \mathrm{Mpc}$ this yields a $3 \sigma$ limit on the luminosity of $L_{v} \lesssim 6.9 \times 10^{40} \mathrm{erg} \mathrm{s}^{-1} \mathrm{~Hz}^{-1}$. This value lies above the peak luminosity level for all but the most X-ray loud SN I at a similar time after explosion (e.g., SN 1998bw; Pian et al. 2000).

\section{LIGHT-CURVE PROPERTIES}

\subsection{Reddening}

Reddening due to the Milky Way in the direction of UGC 2526 has a value of $E(B-V)=0.210 \mathrm{mag}$, according to the IR dust maps of Schlegel et al. (1998). In order to estimate the host-galaxy contribution to the total reddening, we examine our spectra for evidence of narrow $\mathrm{Na}$ I $\mathrm{D}$ lines, which have been shown to correlate with extinction due to dust (Turatto et al. 2003; Poznanski et al. 2012). It is only in our highest signalto-noise ratio spectra (DEIMOS, +9 days) that we see weak $\mathrm{Na}$ I D absorption at the redshift of UGC $2526^{19}$ with an equivalent width $\mathrm{EW}_{\mathrm{Na} \text { ID }} \sim 0.31 \AA$. Using the empirical relation of Poznanski et al. (2012), this implies $E(B-V)_{\text {host }} \approx 0.03$ mag. Given the low level of this inferred effect, combined with the uncertainties in the Na I D relation (e.g., Poznanski et al. 2011), we incorporate this value into our error budget for $E(B-V)_{\text {tot }}$. Throughout this paper we adopt an $R_{V}=A_{V} / E(B-V)=3.1$ Milky Way extinction curve with a total reddening value of $E(B-V)=0.210_{-0.006}^{+0.036} \mathrm{mag}$.

\subsection{Optical Light-curve Evolution}

In the left four panels of Figure 4 we display our BVRI light curves, normalized to peak magnitude and epoch along with the $B V R I$ light curves for other SN I which have previously been referred to as fast or rapidly evolving (black circles). These include the rapid Type Ic SN 1994I (Richmond et al. 1996), the "calcium-rich" Type Ib SN 2005E (Perets et al. 2010), the SN 1991bg-like Type Ia SN 1998de (Modjaz et al. 2001), and the extremely low-luminosity SN 2008ha (Foley et al. 2009, 2010; Valenti et al. 2009). Also shown are SN 2010X (Kasliwal et al. 2010; colored stars), SN 2002bj (Poznanski et al. 2010; colored squares), SN 1885A (de Vaucouleurs \& Corwin 1985; Perets et al. 2011; plus signs), and SN 1939B (Leibundgut et al. 1991; Perets et al. 2011; asterisks).

19 The large Na I D absorption seen in Figure 3 is along the line of site to the galaxy core. 

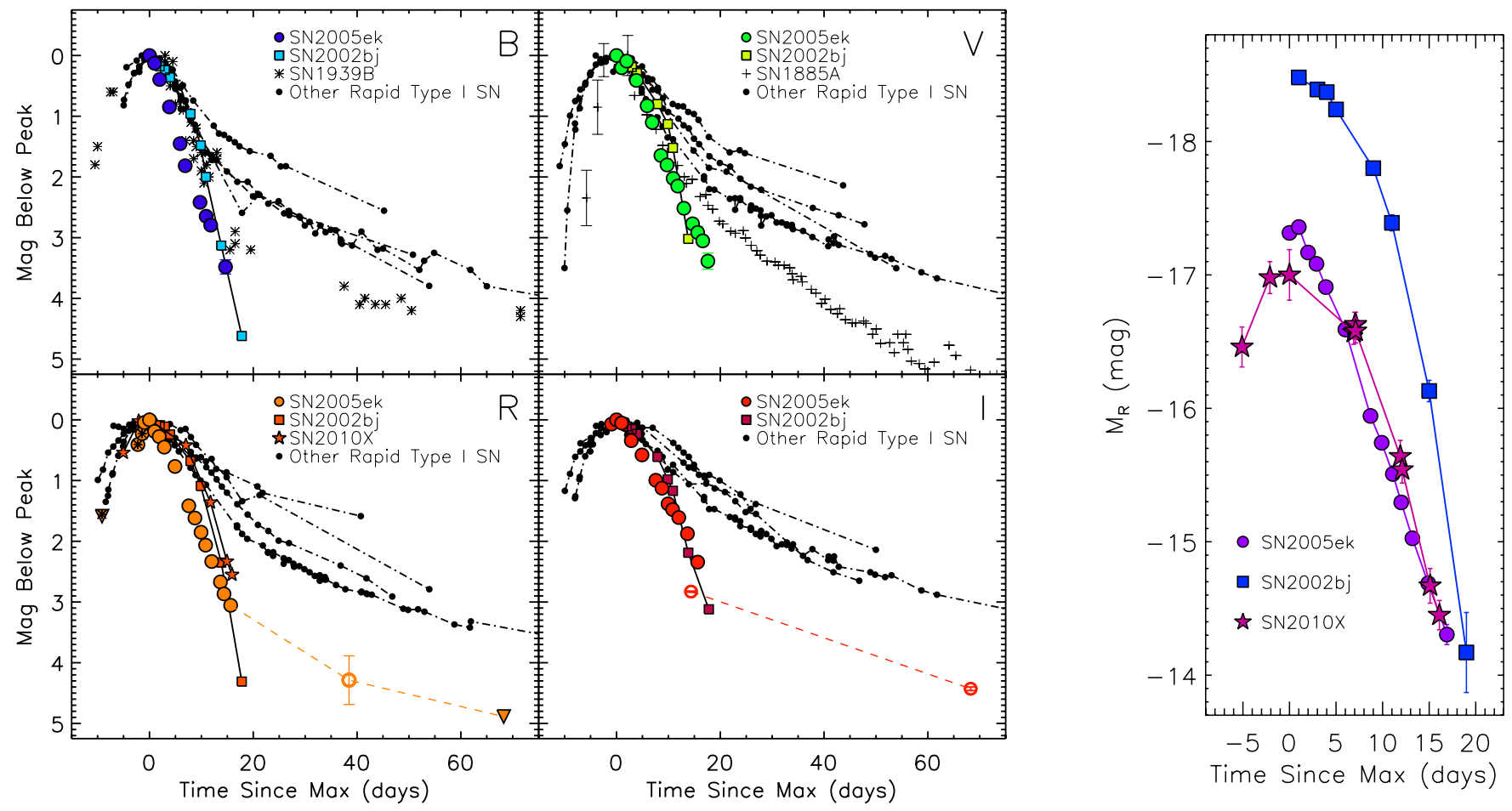

Figure 4. Left: light curve of SN 2005ek (colored circles), normalized to peak magnitude/epoch and compared to other rapidly evolving supernovae of Type I. The other events are SN 1994I, SN 2008ha, SN 1998de, SN 2005E (black circles), SN 2002bj (colored squares), and SN 2010X (colored stars; $R$-band panel only). Right: $R$-band absolute magnitude light curves of SN 2005ek (purple circles), SN 2002bj (blue squares), and SN 2010X (magenta stars). When error bars are not visible they are smaller than the plotted points.

(A color version of this figure is available in the online journal.)

Table 5

Basic Photometric Properties

\begin{tabular}{lccccr}
\hline \hline Band & $\begin{array}{c}m_{\text {obs, max }}{ }^{\mathrm{a}} \\
(\mathrm{mag})\end{array}$ & $\begin{array}{c}M_{\mathrm{abs}, \max }{ }^{\mathrm{b}} \\
(\mathrm{mag})\end{array}$ & $\begin{array}{c}\Delta m_{15} \\
(\mathrm{mag})\end{array}$ & $\begin{array}{c}\text { Decline Rate }^{\mathrm{c}} \\
\left(\text { mag day }^{-1}\right)\end{array}$ & $\begin{array}{c}\tau_{e}{ }^{\mathrm{d}} \\
(\mathrm{day})\end{array}$ \\
\hline$B$ & $18.25(0.02)$ & $-16.72(0.15)$ & $3.51(0.13)$ & $0.24(0.01)$ & $4.7(0.1)$ \\
$V$ & $17.83(0.02)$ & $-16.96(0.15)$ & $2.79(0.07)$ & $0.22(0.01)$ & $6.9(0.1)$ \\
$R$ & $17.41(0.02)$ & $-17.26(0.15)$ & $2.88(0.05)$ & $0.21(0.01)$ & $6.3(0.1)$ \\
$I$ & $17.13(0.04)$ & $-17.38(0.15)$ & $2.13(0.06)$ & $0.15(0.01)$ & $8.5(0.2)$ \\
$J$ & $17.10(0.09)$ & $-17.26(0.17)$ & $1.17(0.50)$ & $0.11(0.01)$ & $9.6(0.5)$ \\
$H$ & $16.85(0.10)$ & $-17.45(0.18)$ & $1.62(0.77)$ & $0.09(0.01)$ & $12.6(0.7)$ \\
$K$ & $16.49(0.10)$ & $-17.78(0.18)$ & $2.51(1.12)$ & $0.09(0.02)$ & $10.2(0.9)$ \\
\hline & & & & &
\end{tabular}

Notes.

a Not corrected for Milky Way extinction.

b Corrected for Milky Way extinction and assuming no host-galaxy extinction.

${ }^{c}$ As measured by a linear fit between +2 and +18 days (from $R$-band maximum).

$\mathrm{d}$ Time for light curve to decline by a factor of $1 / e$.

From Figure 4 it is clear that SN 2005ek is an outlier even among rapid Type I SNe, decaying by $\gtrsim 3 \mathrm{mag}$ in 15 days and showing an unusually linear decline immediately postmaximum. However, our final $r^{\prime}$ and $i^{\prime}$ detections do show evidence for a change in slope around 20-30 days postmaximum. We can place an upper limit of $\sim 0.029 \mathrm{mag}_{\text {day }}{ }^{-1}$ on the late-time $i^{\prime}$-band slope of SN 2005ek by comparing the two P200 $i^{\prime}$ detections. Both the timing of this transition and the late-time slope are comparable to those of the other rapid SN I plotted in Figure 4, although SN 2005ek decays by 1-2 mag more before settling onto this late-time tail.

Basic properties for the BVRIJHK bands are given in Table 5 . We find the $R$-band peak epoch by fitting a low-order polynomial to the P60 R-band light curve supplemented with the Lick unfiltered photometry (which most closely mimics and is calibrated to the $R$ band; Li et al. 2003). This yields a peak epoch (MJD) of $53639.9 \pm 0.3$ day. Unless otherwise noted, all phases throughout this paper are in reference to $R$-band maximum. After correcting for distance and reddening we derive peak absolute $B V R I$ magnitudes ranging from $-16.72 \pm 0.15$ ( $B$ band) to $-17.38 \pm 0.15$ ( $I$ band). This places SN $2005 \mathrm{ek}$ at a peak optical magnitude very similar to SN $2010 \mathrm{X}$ and $\sim 1.5 \mathrm{mag}$ below SN 2002bj, SN 1885A, and SN 1939B. In the right panel of Figure 4 we compare the absolute $R$-band light curves of SN 2005ek, SN 2010X, and SN 2002bj.

In order to quantify the rapid decline of SN 2005ek we calculate the time over which the magnitude declines by a factor of $e^{-1}\left(\tau_{e}\right)$, the number of magnitudes the light curve declines in the first 15 days past maximum $\left(\Delta m_{15}\right)$, and the linear decline rate in magnitudes per day. The first two quantities are calculated by linearly interpolating our data, and are measured with respect to the observed peak magnitude/date. The decline rates are estimated from linear least-square fits to the data between +2 and +16 days. Uncertainties for all properties listed in Table 5 were estimated using a Monte Carlo technique to produce and analyze 1000 realizations of our data. ${ }^{20}$

Our best constraint on the rise time of SN 2005ek comes from the nondetection in a LOSS search image obtained on September 18.5 (only $\sim 9$ days before the observed $R$-band maximum). Despite this relatively short time frame, the upper limit of $\sim 19$ mag only moderately constrains the explosion epoch (Figure 2). We can infer that SN 2005ek rose slightly faster than its initial decline.

\footnotetext{
${ }^{20}$ Each data point in each realization is a random variable chosen from a normal distribution with a mean and variance determined by its counterpart in our initial data set.
} 


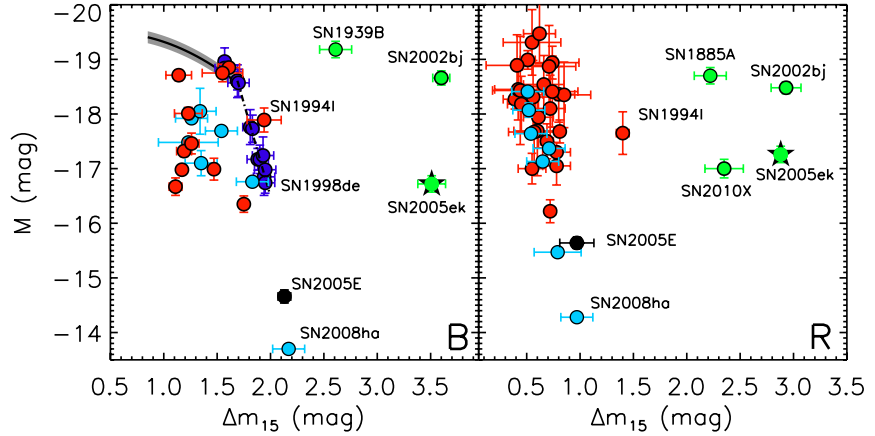

Figure 5. Absolute magnitude vs. $\Delta m_{15}$ for a variety of $\mathrm{SN}$ in the $B$ band (left panel) and $R$ band (right panel). Normal SNe Ia are represented by a black line and shaded region. SN 1991bg-like SNe Ia are shown in dark blue, SNe Iax are shown in light blue, SNe Ib/Ic are shown in red, SN 2005E is shown in black, and SN 2002bj, SN 2010X, SN 1885A, SN 1939B and SN 2005ek are shown in green. SN 2005ek is highlighted by a star. Note the observations obtained for SN $1885 \mathrm{~A}$ are actually closer to modern day $V$-band. Objects plotted in Figure 4 are labeled.

(A color version of this figure is available in the online journal.)

One of the most distinctive features of SN Ia is the tight correlation between light-curve peak magnitude and decay rate. This is in stark contrast to SN Ib/Ic, which have been shown to fill a large portion of this parameter space (Drout et al. 2011). In Figure 5 we plot peak absolute magnitude versus $\Delta m_{15}$ for SN 2005ek and other SNe of Type I.

The left panel of Figure 5 displays these values as measured in the $B$ band, which allows for a comparison with the wellstudied Phillips (1993) relation for normal SNe Ia (Phillips et al. 1999; gray shaded region and solid black line) and the steeper relation found by Taubenberger et al. (2008; dark blue points and dashed black line) for "fast" SNe Ia, as well as SN 1939B. Also shown in this panel are the literature sample of SNe Ib/Ic from Drout et al. (2011; red points), the SNe Iax sample from Foley et al. (2013; light blue points), and several other peculiar Type I events. The right panel, measured in the $R$ band, allows for a comparison to the full sample of $\mathrm{SNe} \mathrm{Ib} / \mathrm{Ic}$ from Drout et al. (2011) as well as SN 2010X (for which $r$ was the only wellsampled band obtained). We also include SN 1885A which was observed in a photographic band which most closely resembles modern day $V$-band. SN 2005ek falls well outside the phase space covered by normal $\mathrm{SNe} \mathrm{Ib} / \mathrm{Ic}$ and is inconsistent with a simple extrapolation of either of the two SN Ia scaling relations.

\subsection{Color Evolution}

In Figure 6 we plot the $B-V, V-R$, and $R-I$ colors for SN 2005ek, along with the color evolution for other rapidly evolving events. Also displayed is the Lira relation (Phillips et al. 1999) which describes the remarkably similar $B-V$ color evolution for SNe Ia between 30 and 90 days past $V$-band maximum (dashed line; top panel). In the middle panel we also show the SN Ib/Ic template color curve from Drout et al. (2011; gray shaded region). Drout et al. (2011) demonstrated that dereddened $\mathrm{SNe} \mathrm{Ib} / \mathrm{Ic}$ show a very similar $V-R$ color evolution with a minimum dispersion at $\sim 10$ days post-maximum.

In the first $\sim 20$ days post-maximum, the $B-V$ and $V-R$ colors of SN 2005ek appear consistent with those of other rapidly evolving $\mathrm{SNe} \mathrm{I}$ : they exhibit a steady reddening with time and tentative evidence for the onset of a plateau between $\sim 10$ and 15 days. Although the $R-I$ colors of SN 2005 ek also show a steady reddening, they do so at a much steeper rate than the other events displayed in Figure 6. Because this unusual

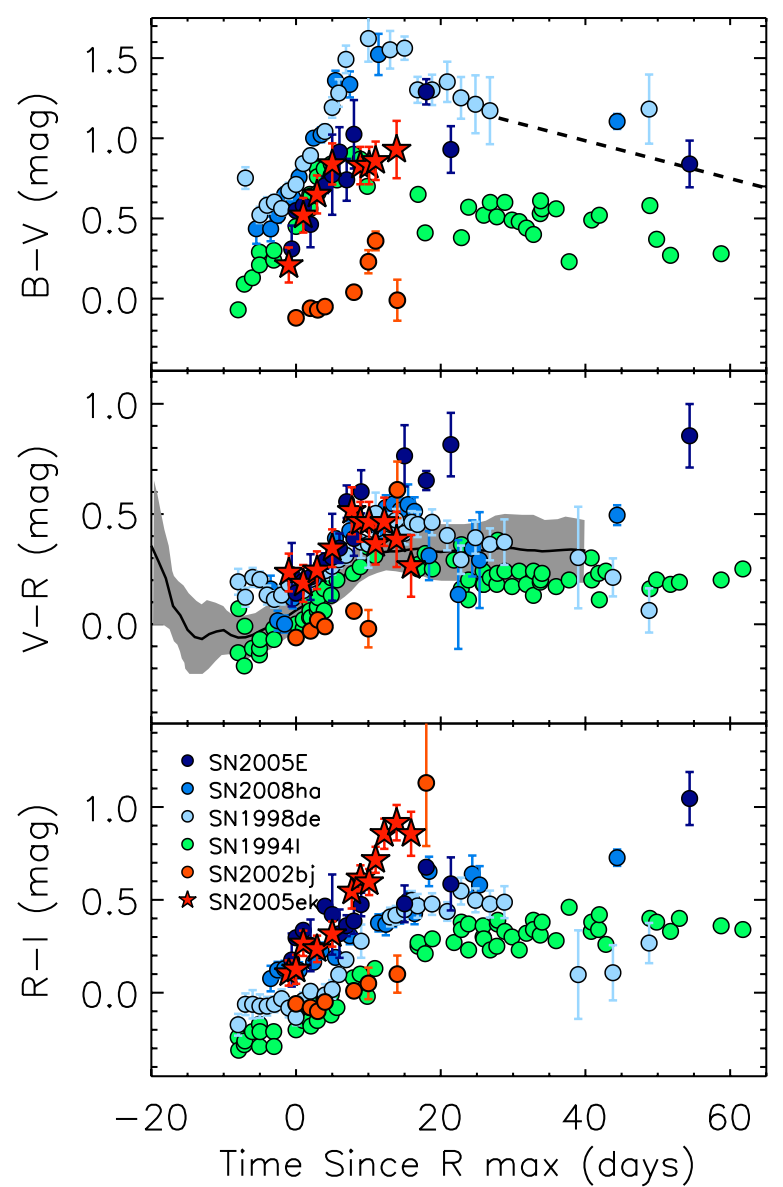

Figure 6. $B-V, V-R$, and $R-I$ color evolution for SN 2005ek (stars) and other rapidly evolving $\mathrm{SNe}$ (circles). See text for details.

(A color version of this figure is available in the online journal.)

evolution is present only in $R-I$, it cannot be fully explained by a rapid cooling of the SN ejecta, but must be caused, in part, by a strong spectroscopic feature. Indeed, from Figure 3 we see that by +9 days the emission component of the Ca II near-IR triplet (which falls solidly inside the $I$ band) has grown substantially.

Although the colors of SN 2005ek are broadly consistent with those of other SNe I, they vary substantially from those of SN 2002bj. SN 2002bj appears much bluer than any other object in $B-V$ and actually shows very little color evolution in either $V-R$ or $R-I$ until the final epoch, when it drastically reddens.

\subsection{Spectral Energy Distribution}

In Figure 7 we plot the UV-optical-IR SED of SN 2005ek from September 30 (3 days post-maximum). Like many SN I near maximum brightness, the SED of SN 2005ek peaks in the optical.

Blackbody fits to various portions of the SED yield temperatures clustered around $7000 \mathrm{~K}$ (Figure 7, red curve). This likely represents a lower limit on the true temperature due to the strong UV line blanketing produced by iron-peak elements in the spectra of SNe I (see, e.g., Mazzali \& Lucy 1993; Bongard et al. 2008). Detailed modeling of the photospheric spectra reveals an ionization temperature of 9000-10,000 K near maximum brightness (Section 4). 


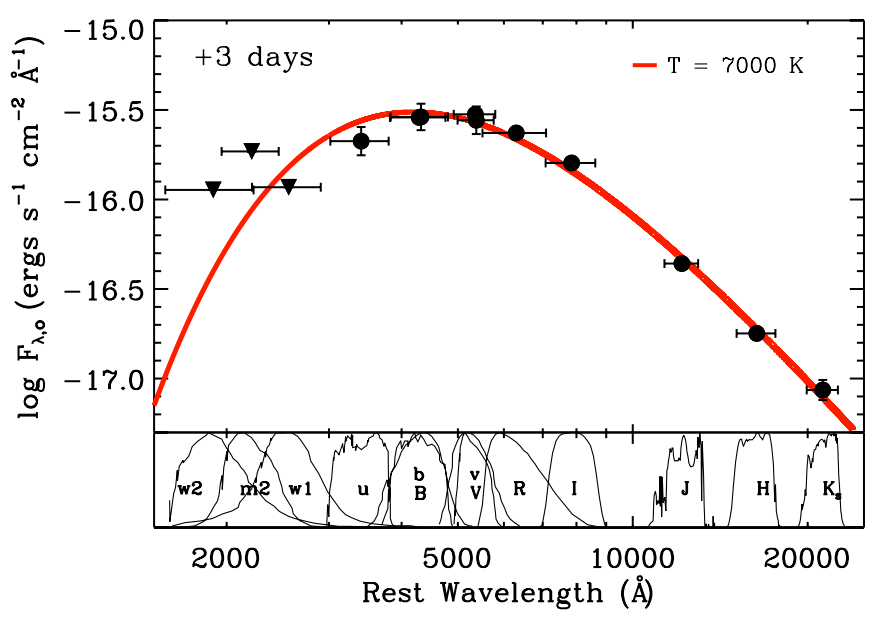

Figure 7. UV through IR spectral energy distribution of SN 2005ek at three days post-maximum. Upper limits are indicated as triangles and bandpass shapes are shown in the lower panel. The best-fitting $7000 \mathrm{~K}$ blackbody is shown as a red line.

(A color version of this figure is available in the online journal.)

\subsection{Pseudo-bolometric Light Curve}

To construct a pseudo-bolometric light curve we sum our observed $B V R I J H K$ data by means of a trapezoidal interpolation and attach a blackbody tail with a temperature and radius found by fitting a Planck function to the data. For epochs where we do not possess $J H K$ data we add a factor to our summed $B V R I$ data such that the total IR contribution ranges from $\sim 20 \%$ near maximum to $\sim 40 \%$ at the end of our observations (Valenti et al. 2007). We do not include a UV correction as our Swift UV observations contain only upper limits. Using this method, we find a peak bolometric luminosity of $(1.2 \pm 0.2) \times 10^{42} \mathrm{erg} \mathrm{s}^{-1}$ and a total radiated energy between -1 and +16 days of $(8.2 \pm 0.3) \times 10^{47} \mathrm{erg}$.

We also use the $r^{\prime}$-band detection at +38 days and the $i^{\prime}$-band detection at +68 days to place constraints on the latetime pseudo-bolometric evolution of SN 2005ek. First, we sum the observed flux at each epoch over the width of the appropriate filter to yield estimates for the minimum bolometric luminosity at +38 and +68 days. Second, by comparing the luminosity contained in our P200 $r^{\prime} i^{\prime}$-band observations at +15 days to our inferred bolometric luminosity at that epoch, we find that at +15 days the $r^{\prime}$ and $i^{\prime}$ bands contained $\sim 15 \%$ and $\sim 14 \%$ of the bolometric luminosity, respectively. We use this fact to estimate a maximum pseudo-bolometric luminosity at +38 and +68 days under the assumption that the $r^{\prime}$ and $i^{\prime}$ contributions to the total luminosity will continue to increase. The true bolometric luminosity likely lies closer to the higher of these two constraints.

In Figure 8 we plot our pseudo-bolometric light curve of SN 2005ek (red stars) along with the pseudo-bolometric light curves of SN 1994I (Richmond et al. 1996), SN 2008ha (Moriya et al. 2010), SN 2002bj (Poznanski et al. 2010), SN 2010X (Kasliwal et al. 2010), SN 2005E, and SN 1998de. These last two were constructed in the manner described above from the photometry of Perets et al. (2010) and Modjaz et al. (2001). The bolometric curve of SN 2010X was constructed by computing $v F_{v}$ in the $r$ band. A similar analysis of SN 2005ek yields a pseudo-bolometric curve which is broadly consistent with our analysis above, but declines slightly more rapidly. The hatched region in Figure 8 represents our constraints on the late-time pseudo-bolometric evolution of SN 2005ek.

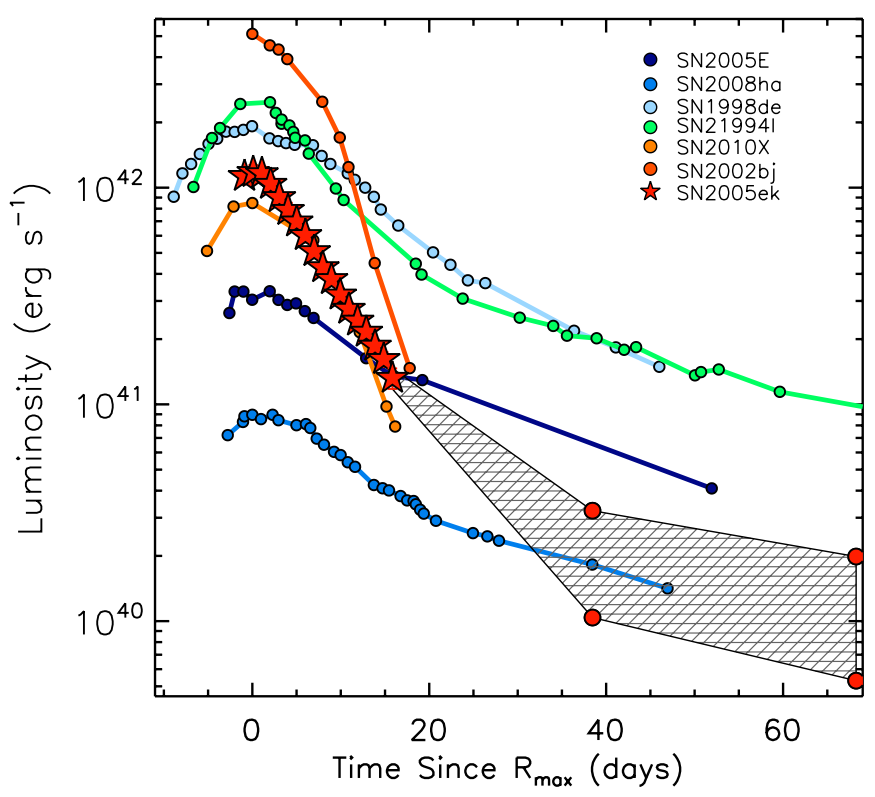

Figure 8. Pseudo-bolometric light curve for SN 2005ek (red stars) along with those of several other rapidly evolving supernovae (circles). The red circles show our constraints on the late-time pseudo-bolometric luminosity of SN 2005ek based on single-band detections. The hatched region is meant to guide the eye. (A color version of this figure is available in the online journal.)

\section{SPECTROSCOPIC PROPERTIES}

The spectra of SN 2005ek shown in Figure 3 show considerable evolution over a short time period, and most closely resemble those of normal SNe Ic. In Figure 9 we compare the maximum-light and transitional ${ }^{21}$ spectra of SN $2005 \mathrm{ek}$ to a set of SNe Ic (SN 2010X, SN 1994I, SN 2007gr, and SN 2004aw). SN 2005ek reaches the transitional phase much faster than the other events but the spectroscopic similarities at both epochs are clear. Of the events displayed in Figure 9, only SN 2010X decays on a timescale similar to that of SN 2005ek. The others possess $\Delta m_{15, R}$ values ranging from $\sim 1.4$ (SN 1994I) to $\sim 0.4$ (SN 2004aw).

In this section, we examine the spectroscopic properties and evolution of SN 2005ek utilizing two modeling techniques. Initial line identifications and estimates of photospheric velocities were made with the spectral synthesis code SYN++ (Thomas et al. 2011). ${ }^{22}$ This analysis offers information about the ions present in a particular ionization state and spectral range, allowing one to cover a large parameter space with minimal time and computational resources. In addition, we model a subset of the spectra using a one-dimensional Monte Carlo radiative transport code developed for SN outflows (Mazzali \& Lucy 1993; Lucy 1999; Mazzali 2000), which allows quantitative assessments of ion abundances to be made. Using the results of both techniques, we compare several distinctive spectroscopic features of SN 2005ek to those of other SNe I and comment on the consequences for explosive nucleosynthesis.

\subsection{SYN++Evolution and Photospheric Velocities}

$\mathrm{SYN}++$ is a parameterized spectral synthesis code which allows empirical fitting of SN spectra without a priori assumptions

\footnotetext{
21 "Transitional" refers to the transition from optically thick to thin, which, in this case, we characterize by an increased prominence of the emission component of the Ca II near-IR triplet).

22 This is an updated version of SYNOW; https://c3.lbl.gov/es/.
} 

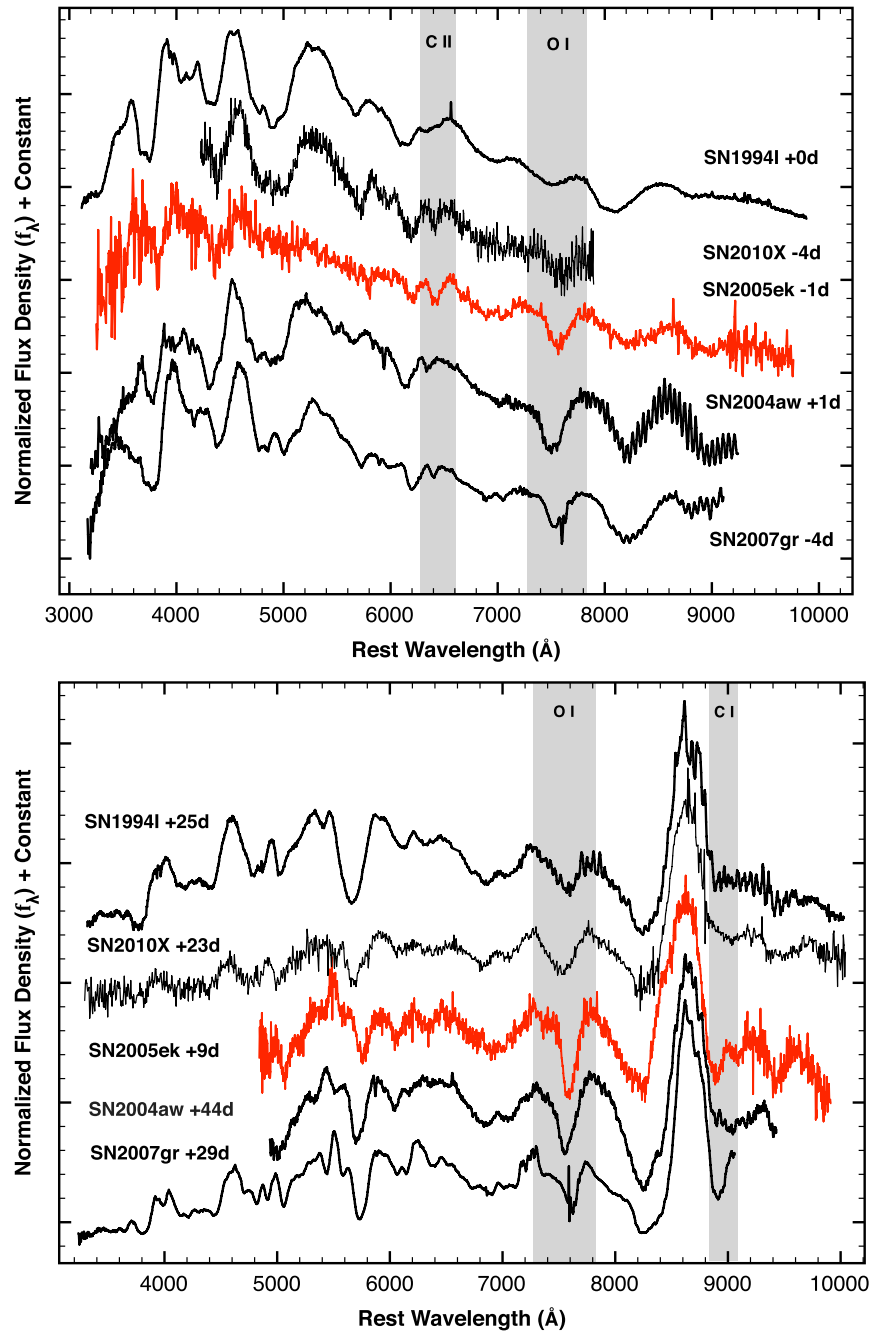

Figure 9. Comparison of SN 2005ek (red) and other well-studied SNe Ic (black; SN 1994I, SN 2004aw, SN 2007gr, and SN 2010X). Strong similarities are seen. Top: near maximum light. The regions around C II $\lambda 6582$ and O I $\lambda 7774$ are shaded. Bottom: transitional spectra. The region around $\mathrm{O}_{\mathrm{I}} \lambda 7774$ and $\mathrm{C}$ I $\lambda 9095$ are shaded.

(A color version of this figure is available in the online journal.)

of the ejecta's density and composition structure. It operates under the assumption of spherical symmetry, homologous expansion (radius proportional to velocity), a sharp photosphere, and a pseudo-blackbody continuum level. Line formation is due to pure resonant scattering (treated using the Sobolev approximation) and Boltzmann statistics are utilized to determine the relative line strengths for a given ion. For more details, see Branch et al. (2002) and Thomas et al. (2011).

In Figure 10 we show three representative SYN++ fits, covering the evolution of SN $2005 \mathrm{ek}$ between -1 and +9 days. Major spectroscopic features are labeled. In all cases, the excitation temperature was set to $10,000 \mathrm{~K}$ and we chose an exponential form for the optical depth profile. The photospheric velocity used in these fits ranges from $\sim 9000 \mathrm{~km} \mathrm{~s}^{-1}$ (day -1) to $\sim 7000 \mathrm{~km} \mathrm{~s}^{-1}$ (day +9 ).

The near-maximum-light spectra of SN 2005ek can be modeled with a combination of O I, C II, Mg II, Si II, Ca II, Ti II, and some Fe II at $8000-9000 \mathrm{~km} \mathrm{~s}^{-1}$. S II is also included, although evidence for it is very weak. Between the -1 day and maximumlight models Na I was added to describe the feature near $5700 \AA$. In Figure 11 we present the individual ion components of the

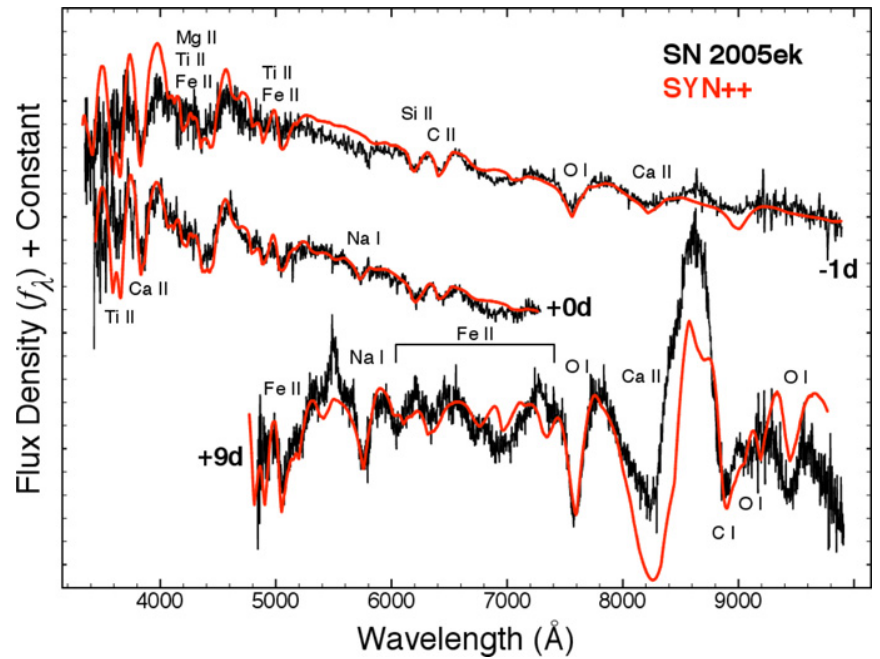

Figure 10. SYN++ model fits to the -1 day, maximum-light, and +9 day spectra of SN 2005ek (red lines). Observed spectra are shown in black. Major spectroscopic features are labeled.

(A color version of this figure is available in the online journal.)

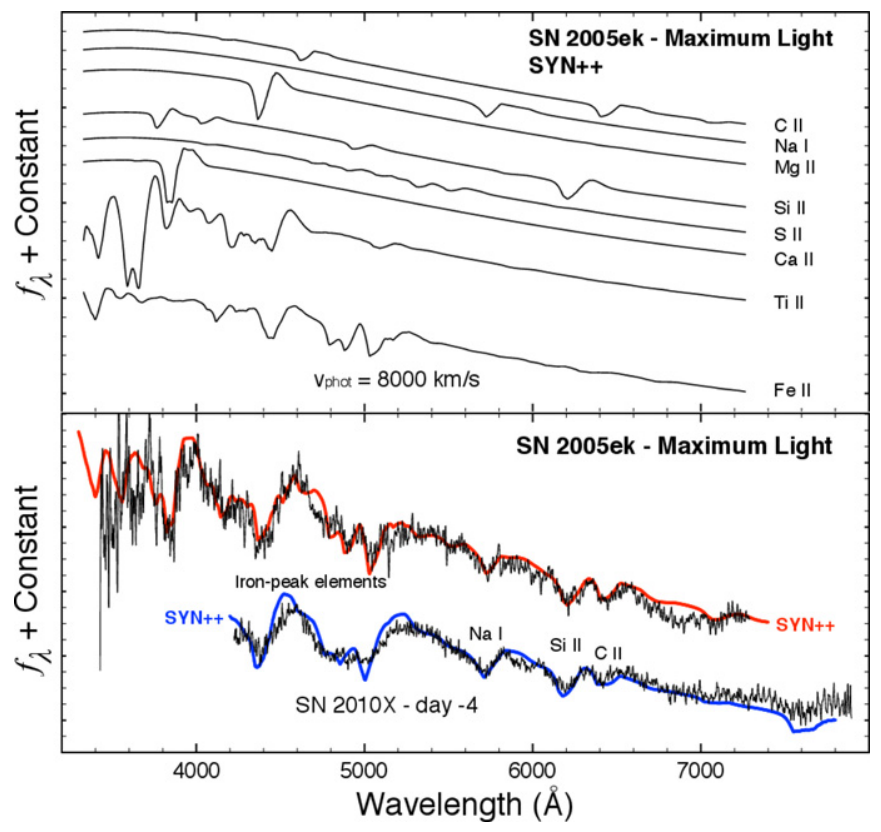

Figure 11. Top: SYN++ model for the maximum-light spectrum of SN 2005ek, separated by ion. Bottom: full model (red) along with a similar model constructed for the -4 day spectrum of SN 2010X (blue). Observed spectra are shown in black.

(A color version of this figure is available in the online journal.)

maximum-light model. Also displayed (blue, lower panel) is a model constructed from the same set of ions for the -4 day spectrum of SN 2010X. This highlights the similarities between the spectra of SN 2005ek and SN 2010X and demonstrates that our fitting scheme is equally applicable to both events.

Despite growth in the emission component of the Ca II nearIR triplet, the +9 day spectrum of SN 2005ek still shows a partial environment of resonant-line scattering, indicating it can be approximately modeled with SYN++. By this epoch, the Si II and $\mathrm{C}$ II features found near maximum light have already faded. A majority of the features can be attributed to $\mathrm{Fe}$ II and $\mathrm{O}$ I, along with Na I, Ca II, and a decent fit to C I 29095 (consistent with the presence of $\mathrm{C}$ II at earlier epochs) at $\sim 7000 \mathrm{~km} \mathrm{~s}^{-1}$. SYN++ fits 
to the intermediate epochs are consistent with the decrease in velocity, increase in prominence of Fe II and $\mathrm{Ti}$ II features, and decrease in prominence of the $\mathrm{C}$ II and $\mathrm{Si}$ II features between the maximum-light and +9 day spectra.

\subsection{Abundance Modeling}

To obtain quantitative estimates of the elemental abundances present in the ejecta of SN $2005 \mathrm{ek}$, we also model the -1 day, maximum-light, and +9 day spectra with the one-dimensional Monte Carlo radiation transport code described by Mazzali \& Lucy (1993), Lucy (1999), and Mazzali (2000). The code assumes spherical symmetry and that radiation is emitted as a blackbody at a lower boundary (a pseudo-photosphere). The SN ejecta are defined by a run of density versus velocity (an "explosion model") and a depth-dependent set of abundances. Energy packets are allowed to interact with the ejecta gas via excitation processes and electron scattering. The state of the gas is computed according to that of the radiation using a lambda iteration and adopting the modified nebular approximation (Mazzali \& Lucy 1993), while the emerging spectrum is computed by formally solving the transfer equation in a final step (Lucy 1999; Mazzali 2000). This method has been successfully applied to both SNe Ia (e.g., Mazzali et al. 2008) and SNe Ib/Ic (e.g., Sauer et al. 2006).

In order to model the rapidly evolving spectra of SN $2005 \mathrm{ek}$ we first had to establish a reasonable explosion model. The fast light curve and moderate velocities indicate a small ejecta mass. We experimented with different possibilities, and found that a model with mass $\sim 0.3 M_{\odot}$ and $E_{K} \approx 2.5 \times 10^{50} \mathrm{erg}$ provides a reasonable match to the evolution of the spectra. The distribution of density with velocity resembles a scaled-down W7 model (Nomoto et al. 1984).

Using this model, and assuming a rise time of 14 days, we were able to reproduce the spectroscopic evolution of SN 2005ek (Figure 12). As the photosphere recedes inward, each model epoch constrains a larger amount of the ejected material. With spectral coverage out to +9 days we are able to probe the outer $\sim 0.2 M_{\odot}(\sim 66 \%)$ of the ejecta.

The results are qualitatively consistent with the SYN++ line identifications given above. However, abundances do not necessarily correlate with line strength. Notably, the strength of the $\mathrm{O}$ I $\lambda 7774$ line requires a high oxygen abundance, so that the composition is dominated by oxygen $(\sim 80 \%)$. Fe is present and is responsible for the absorption near $5000 \AA$, while Ti and Cr are also important for shaping the spectrum near $4000 \AA$, but small abundances are sufficient for this as well as for $\mathrm{Ca}$, despite the strength of the near-IR triplet absorption. Smaller fractions of $\mathrm{Mg}, \mathrm{C}$, and Si are also present; $\mathrm{Mg}$ II lines leave a strong imprint in the earlier spectra, especially in the red (features at $8900 \AA$ and $7600 \AA$, the latter in a blend with the stronger O I line), but also in the blue ( $4481 \AA$, which is the stronger contributor to the absorption near $4000 \AA$ ).

The composition shows little or no variation between the three epochs. The ejecta are dominated by oxygen $(\sim 86 \%)$, with intermediate-mass elements such as $\mathrm{C}, \mathrm{Mg}$, $\mathrm{Si}$, and limited amounts of $\mathrm{S}$ and $\mathrm{Ca}$ contributing $\sim 13.5 \%$ and iron-peak elements (Fe, $\mathrm{Ni}, \mathrm{Cr}, \mathrm{Co}, \mathrm{Ti})$ contributing only $\sim 0.5 \%$.

\subsection{Notable Spectroscopic Features}

The ions utilized in the models above are typical of SNe I. However, several features warrant further discussion in the context of SN 2005ek.

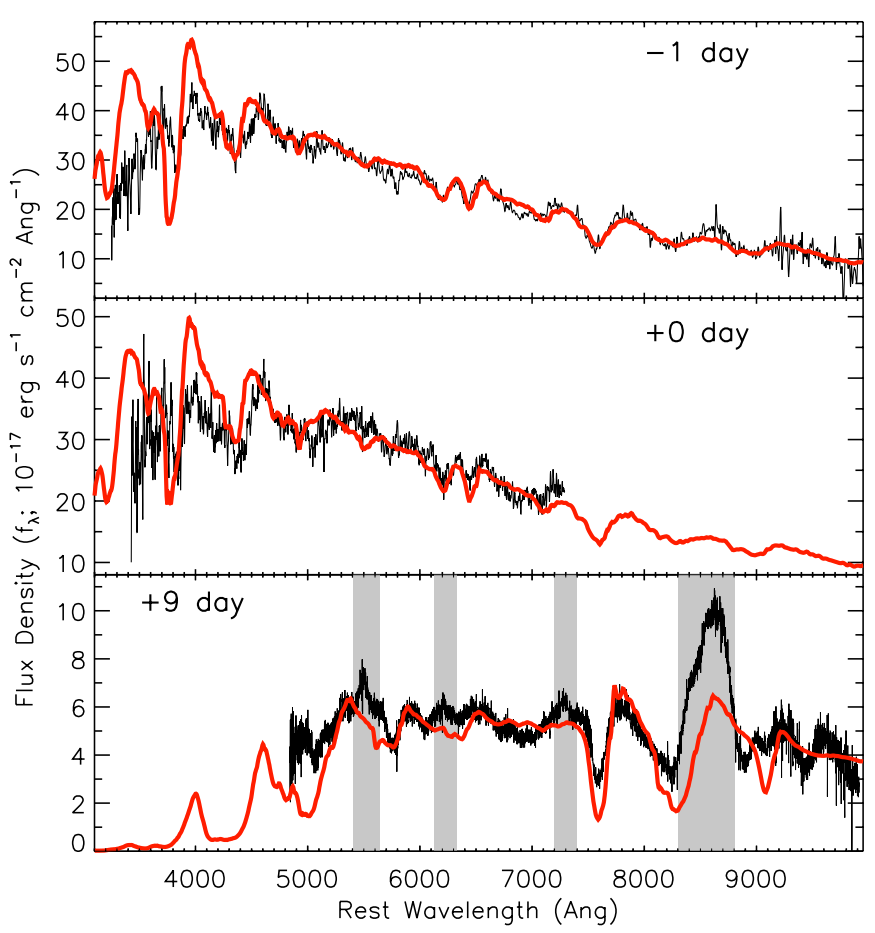

Figure 12. One-dimensional Monte Carlo radiation transport models for the -1 day (top panel), maximum-light (middle panel), and +9 day (lower panel) spectra of SN 2005ek. Observed spectra are shown in black, models in red. Regions showing excess with respect to the +9 day model, which may represent the onset of nebular features, are shaded (gray).

(A color version of this figure is available in the online journal.)

\subsubsection{Carbon Features}

The C II $\lambda 6582$ feature near $6400 \AA$ is noticeably prominent in the near-maximum-light spectra of SN 2005ek. Its strength is comparable to the Si II $\lambda 6355$ feature. This is due in part to the relative weakness of the $\mathrm{Si}$ II feature, which, in the onedimensional Monte Carlo models presented above, is caused by a combination of the low overall Si abundance $(\sim 2 \%)$ and the relatively low-density environment. However, the strength of the $\mathrm{C}$ II feature is unusual in its own right, and it is still present several days post-maximum (Figure 3 ).

In $\mathrm{SNe}$ Ia, carbon features trace the distribution of unburned material from the carbon-oxygen $(\mathrm{C}-\mathrm{O})$ WD. $\mathrm{C}$ II features are observed in $\sim 30 \%$ of $\mathrm{SNe}$ Ia although they are rarely either this strong or after maximum light (Howell et al. 2006; Thomas et al. 2007; Parrent et al. 2011; Silverman \& Filippenko 2012; Folatelli et al. 2012). In general, the detection of carbon is also considered to be unusual in SNe Ic. However, all the spectra shown in Figure 9 also show evidence for a notch in this region (highlighted in gray), exemplifying the spectroscopic similarity of these objects. This feature was specifically identified as C II in SN 2007gr (Valenti et al. 2008) and SN 2004aw (Taubenberger et al. 2006), while it is more debated in SN 1994I (Wheeler et al. 1994) where features are broader and, hence, more blended. In the bottom panel of Figure 9, we also highlight the region around the feature we identify as $\mathrm{C}_{\mathrm{I}} \lambda$ 2095 . A similar notch is seen in SN 2007gr. Note that despite the strength of the C II feature, the inferred carbon to oxygen abundance ratio is still small $(\sim 0.02)$.

\subsubsection{Iron-peak Features}

In the -1 day spectrum of SN 2005ek the broad feature between $4600 \AA$ and $5200 \AA$, attributed mainly to Fe II, is noticeably weaker than in the pre-maximum-light spectra of 


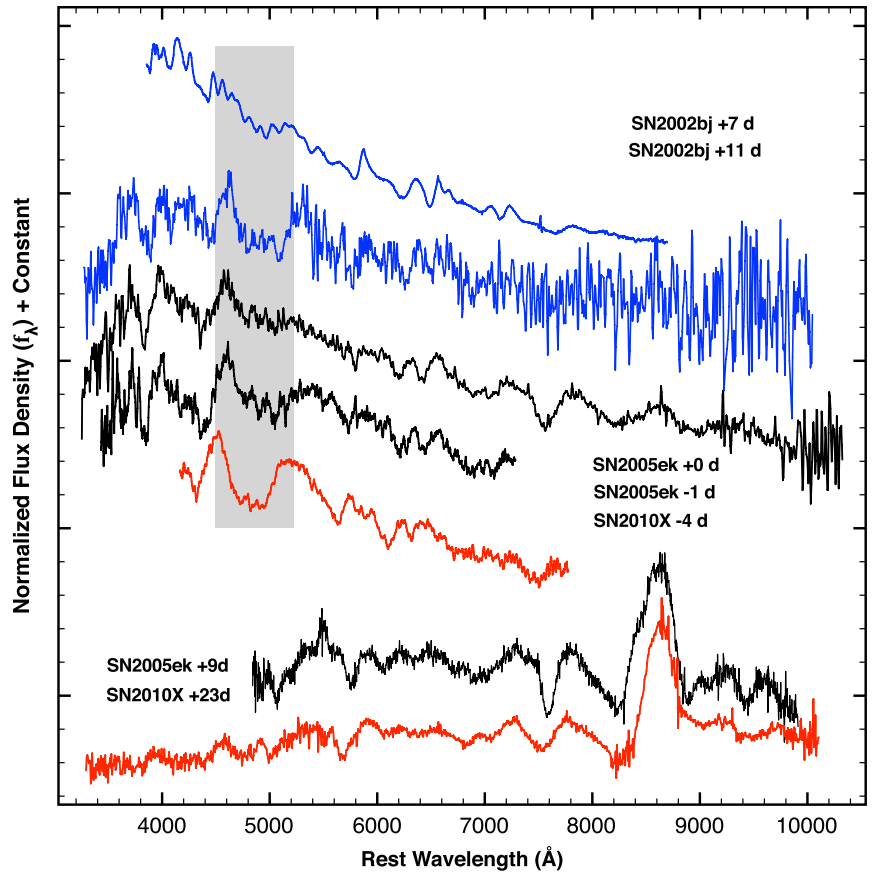

Figure 13. Comparison of SN 2005ek, SN 2010X, and SN 2002bj spectra. Top: "early"-time spectra. Bottom: "late"-time spectra. No spectra from a similar phase are available for SN 2002bj.

(A color version of this figure is available in the online journal.)

other SNe Ic (top panel of Figure 9). The depth of the feature does increase in later epochs (Figures 3 and 13). Attempting to replicate this feature with other iron-peak elements produces less satisfactory model spectra.

The presence of a strong Ti II feature around $4400 \AA$ is one of the distinctive spectroscopic features of the rapidly declining $\mathrm{SN}$ 1991bg-like SNe Ia. Although Ti II is also identified in the models above, the lack of a strong Si II absorption line (another distinctive feature of SN 1991bg-like SN) distinguishes SN 2005ek from these events.

\subsubsection{Helium Features}

None of the models presented above include helium. However, although its presence is not required, our data do not completely rule out its presence. Due to the similarity in wavelength of $\mathrm{He}_{\mathrm{I}}(\lambda 5875, \lambda 6678)$ to $\mathrm{Na} \mathrm{I} \lambda 5890$ and $\mathrm{C}_{\mathrm{II}} \lambda 6582$, some ambiguity between ions can result (e.g., Kasliwal et al. 2010). In our case, the lack of features between $5200 \AA$ and $5800 \AA$ in the -1 day spectrum (see Figure 9) would require any such helium features to have increased in strength after maximum light. This would not be inconsistent with our abundance models, where we required an increase in sodium abundance to model the $\mathrm{Na}$ I feature in the +9 day spectrum. However, He I is difficult to excite (Mazzali \& Lucy 1998; Dessart et al. 2012), especially in relatively cool radiation fields like those of SN 2005ek, and we have no direct evidence for helium emission. We find that the lack of helium in our fits does not require the presence of aluminum as implied by Kasliwal et al. (2010). Rather, a majority of the SN features can be reproduced with a combination of Fe II and $\mathrm{NaI}$.

\subsubsection{Nebular Features}

The +9 day spectrum of SN 2005ek appears to be transitional between the photospheric and nebular phases, with the Ca II
near-IR feature significantly influenced by net emission. In addition, there are slight excesses with respect to both the SYN++ and Monte Carlo models near $5500 \AA, 6300 \AA$, and $7300 \AA$ ( shaded regions, bottom panel, Figure 12) which may be due to the emergence of [O I] $\lambda 5577,\left[\mathrm{O}_{\mathrm{I}}\right] \lambda \lambda 6300,6364$, and [Ca II] $\lambda \lambda 7291,7324$ in emission. This may represent the earliest onset of nebular features in an SN I observed to date.

\subsection{Comparison to $S N 2002 b j$}

The decline rate of SN 2005ek is most closely matched by SN 2002bj and SN 2010X and in Figure 13 we compare the spectra of these three objects. While SN 2010X possesses very similar spectroscopic features at both early and late times (with moderately higher photospheric velocities), the spectroscopic similarities between SN 2005ek and SN 2002bj are less clear.

Poznanski et al. (2010) model SN 2002bj with SYNOW, finding that a majority of features can be fit with intermediatemass elements $(\mathrm{C}, \mathrm{Si}, \mathrm{S})$ and helium at the relatively low velocity of $\sim 4000 \mathrm{~km} \mathrm{~s}^{-1}$. Notably absent from their fit were any iron-peak elements. This is in stark contrast to the maximum-light spectra of SN 2005ek where both iron and titanium play a significant role, and sulfur is not required. Indeed, the first spectrum of SN 2002bj (top, Figure 13) shows a significantly bluer continuum than any of the spectra obtained for either SN 2005ek or SN 2010X. This spectrum was obtained seven days post-maximum. By +11 days (when a second, lower quality, spectrum was obtained), SN 2002bj appears to show a distinctly different morphology in the blue (Figure 13). The continuum is more depressed in the region between $3000 \AA$ and $5500 \AA$, with features that appear similar to those attributed to iron-peak elements in SN 2005ek. This spectroscopic evolution is clearly distinct from that of SN 2005ek and SN 2010X.

\subsection{Consequences for Nucleosynthesis}

The ejecta of SN 2005ek are dominated by oxygen. Unlike the "calcium-rich" objects, such as SN 2005E whose ejecta was $\sim 50 \%$ calcium (Perets et al. 2010), this does not necessarily imply an unusual nucleosynthetic channel. Oxygen-dominated ejecta are common in models of SN Ic which are due to core collapse in a stripped $\mathrm{C}-\mathrm{O}$ star. In that situation, the large oxygen abundance is due to a combination of the initial composition along with partial carbon burning. In contrast, producing oxygen-dominated ejecta via primarily helium burning (the mechanism invoked in the ".Ia" scenario) is not straightforward. Perets et al. (2010) use a one-zone model to examine the explosive nucleosynthetic outputs of $\mathrm{He}, \mathrm{C}$, and $\mathrm{O}$ mixtures at several temperatures. None of their helium-dominated trials yield oxygen-dominated ejecta.

\section{POWER SOURCE AND EXPLOSION PARAMETERS}

The optical light curves of normal $\mathrm{SNe} \mathrm{Ia}$ and $\mathrm{SNe} \mathrm{Ib} / \mathrm{Ic}$ are powered mainly by the radioactive decay of ${ }^{56} \mathrm{Ni}$. For SN 2005ek, the change in light-curve slope between +20 and +40 days (Figure 8 ) points to radioactive decay as a possible power source. However, the initial decay timescale as well as the almost linear post-maximum decline are unusual. In this section we examine whether a ${ }^{56} \mathrm{Ni}$ power source is consistent with the observations of SN 2005ek described above.

For the purposes of simplified analytic models, the bolometric light curves of $\mathrm{SNe} \mathrm{Ib} / \mathrm{Ic}$ are usually divided into two regimes as follows. (1) The photospheric phase, when the optical depth is high and the shape of the light curve is dependent both on the 


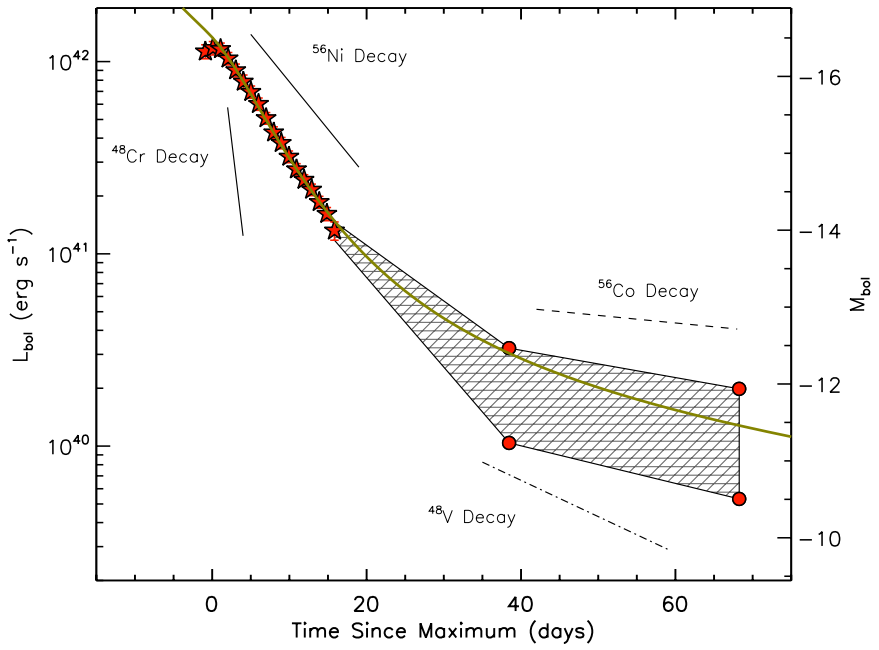

Figure 14. Radioactive models for the pseudo-bolometric light curve of SN 2005ek. Black lines show the decay rates for ${ }^{56} \mathrm{Ni},{ }^{56} \mathrm{Co},{ }^{48} \mathrm{Cr}$, and ${ }^{48} \mathrm{~V}$, assuming full trapping of gamma-rays. The gold curve shows the best-fit model described in the text, assuming $\sim 0.03 M_{\odot}$ of ${ }^{56} \mathrm{Ni}$, a luminosity which tracks the instantaneous energy input, and incomplete gamma-ray trapping.

(A color version of this figure is available in the online journal.)

rate of energy deposition and the photon diffusion time scale (Arnett 1982). In normal $\mathrm{SNe} \mathrm{Ib} / \mathrm{Ic}$, the effects of radiative transfer result in an initial post-maximum decline rate which is slower than ${ }^{56} \mathrm{Ni} \rightarrow{ }^{56} \mathrm{Co}$ decay (Drout et al. 2011). (2) The nebular phase, when the optical depth has decreased and the SN luminosity is determined by the instantaneous rate of energy deposition. Normal $\mathrm{SNe} \mathrm{Ib} / \mathrm{Ic}$ enter this stage at a late epoch ( $\gtrsim 60$ days; Valenti et al. 2007) when the dominant energy source is expected to be ${ }^{56} \mathrm{Co} \rightarrow{ }^{56} \mathrm{Fe}$ decay. The late-time slope of $\mathrm{SN} \mathrm{Ib/Ic} \mathrm{light} \mathrm{curves} \mathrm{are} \mathrm{well} \mathrm{matched} \mathrm{by} \mathrm{this} \mathrm{decay}$ rate when the effects of incomplete gamma-ray trapping are included (Clocchiatti \& Wheeler 1997; Valenti et al. 2007).

The time when an SN will transition to the second of these two phases is determined in large part by the total ejecta mass and kinetic energy of the explosion. In SN $2005 \mathrm{ek}$, both the small inferred ejecta mass $\left(\sim 0.3 M_{\odot}\right)$ and the early onset of nebular spectroscopic features indicate that the assumption of optically thick ejecta may break down within a few days of maximum light, making the models of Arnett (1982) inapplicable. Further, we note that the early portion of the pseudo-bolometric light curve appears linear, and decays at a rate of $0.15 \mathrm{mag} \mathrm{day}^{-1}$, comparable to the 0.12 mag day ${ }^{-1}$ given by ${ }^{56} \mathrm{Ni} \rightarrow{ }^{56} \mathrm{Co}$ decay. In Figure 14 we again plot the pseudo-bolometric light curve of SN 2005ek. Also shown are lines which describe the decay rate of ${ }^{56} \mathrm{Ni} \rightarrow{ }^{56} \mathrm{Co}$ and ${ }^{56} \mathrm{Co} \rightarrow{ }^{56} \mathrm{Fe}$.

With this early decay rate as motivation, we construct a model for the entire post-maximum pseudo-bolometric light curve of SN 2005ek based on the instantaneous rate of energy deposition from the ${ }^{56} \mathrm{Ni} \rightarrow{ }^{56} \mathrm{Co} \rightarrow{ }^{56} \mathrm{Fe}$ decay chain. The model is similar to the nebular phase model of Valenti et al. (2007), although we allow for incomplete trapping of the gamma-rays produced from ${ }^{56} \mathrm{Ni} \rightarrow{ }^{56} \mathrm{Co}$ decay. One effect of incomplete trapping at this early phase is that the light curve should decay by a larger number of magnitudes before settling onto the ${ }^{56} \mathrm{Co}$ tail. This prediction is in good agreement with our comparison of SN 2005ek to other SN I in Section 3.2 (see Figure 4).

Under these assumptions, the luminosity of the SN can be modeled as (Valenti et al. 2007; Sutherland \& Wheeler 1984;
Cappellaro et al. 1997; we use the notation of Valenti et al. 2007)

$$
L(t)=S^{\mathrm{Ni}}(\gamma)+S^{\mathrm{Co}}(\gamma)+S_{e^{+}}^{\mathrm{Co}}(\gamma)+S_{e^{+}}^{\mathrm{Co}}(\mathrm{KE}),
$$

where the four terms describe the energy due to gamma-rays from nickel decay, gamma-rays from cobalt decay, gamma-rays from the annihilation of positrons created in cobalt decay, and the kinetic energy of positrons created in cobalt decay, respectively. These are given by

$$
\begin{aligned}
S^{\mathrm{Ni}}(\gamma) & =M_{\mathrm{Ni}} \epsilon_{\mathrm{Ni}} e^{-t / \tau_{\mathrm{Ni}}}\left(1-e^{-F / t^{2}}\right) \\
S^{\mathrm{Co}}(\gamma) & =0.81 \times \mathcal{E}_{\mathrm{Co}}\left(1-e^{-(F / t)^{2}}\right) \\
S_{e^{+}}^{\mathrm{Co}}(\gamma) & =0.164 \times \mathcal{E}_{\mathrm{Co}}\left(1-e^{-(F / t)^{2}}\right)\left(1-e^{-(G / t)^{2}}\right) \\
S_{e^{+}}^{\mathrm{Co}}(\mathrm{KE}) & =0.036 \times \mathcal{E}_{\mathrm{Co}}\left(1-e^{-(G / t)^{2}}\right),
\end{aligned}
$$

where

$$
\mathcal{E}_{\mathrm{Co}}=M_{\mathrm{Ni}} \epsilon_{\mathrm{Co}}\left(e^{-t / \tau_{\mathrm{Co}}}-e^{-t / \tau_{\mathrm{Ni}}}\right) .
$$

The incomplete trapping of gamma-rays and positrons is incorporated with the terms $\left(1-e^{-(F / t)^{2}}\right)$ and $\left(1-e^{-(G / t)^{2}}\right)$. $F$ and $G$ are constants such that the gamma-ray and positron optical depths decrease by a factor proportional to $t^{-2}$ as expected for an explosion in homologous expansion (Clocchiatti $\&$ Wheeler 1997) and are functions of the total ejecta mass, kinetic energy, and density distribution of the ejecta (Clocchiatti $\&$ Wheeler 1997). Using this model with $M_{\mathrm{Ni}}=0.03 M_{\odot}$, $F=12.8$ days, and $G \approx 16.1 F$ (Valenti et al. 2007), we find the gold curve shown in Figure 14 which matches the early decay rate of SN 2005ek and is also consistent with our latetime constraints. Adopting the parameterization of Valenti et al. (2007) where $F \approx 32 M_{\mathrm{ej}, \odot} / \sqrt{E_{K, 51}}$, this value of $F$ implies a $M_{\mathrm{ej}, \odot} / \sqrt{E_{K, 51}} \approx 0.4$. Using the observed photospheric velocity near maximum $\left(\sim 8500 \mathrm{~km} \mathrm{~s}^{-1}\right)$, we can break the degeneracy between $M_{\mathrm{ej}}$ and $E_{K}$ to yield explosion parameters of $M_{\mathrm{ej}} \approx$ $0.7 M_{\odot}$ and $E_{K} \approx 5.2 \times 10^{50} \mathrm{erg}$. These values are a factor of two larger than those used in our spectroscopic modeling $\left(M_{\mathrm{ej}}=0.3 M_{\odot}\right.$ and $E_{K}=2.5 \times 10^{50} \mathrm{erg}$; Section 4.2), but given the number of assumptions required to extract explosion parameters from this simplified analytic model the two are relatively consistent. We adopt conservative estimates of the explosion parameters to be $M_{\mathrm{ej}}=0.3-0.7 M_{\odot}$ and $E_{K}=$ $2.5-5.2 \times 10^{50} \mathrm{erg}$.

It has been suggested (e.g., Shen et al. 2010) that other radioactive decay chains such as ${ }^{48} \mathrm{Cr} \rightarrow{ }^{48} \mathrm{~V} \rightarrow{ }^{48} \mathrm{Ti}$, which possess shorter decay times than ${ }^{56} \mathrm{Ni} \rightarrow{ }^{56} \mathrm{Co} \rightarrow{ }^{56} \mathrm{Fe}$, may contribute to the luminosity of rapidly evolving events. In Figure 14 we also include lines that represent the decay rates of ${ }^{48} \mathrm{Cr} \rightarrow{ }^{48} \mathrm{~V}$ and ${ }^{48} \mathrm{~V} \rightarrow{ }^{48} \mathrm{Ti}$. The rapid ${ }^{48} \mathrm{Cr}$ decay time $\left(\tau_{\mathrm{Cr}}=1.3\right.$ days $)$ implies that by a few days post-explosion the power input should already be dominated by ${ }^{48} \mathrm{~V} \rightarrow{ }^{48} \mathrm{Ti}$ decay. Although photon diffusion likely plays a role at very early times, it is difficult to reconcile this power source with the change in light-curve slope observed between +20 and +40 days. In addition, in order to fit both of our late-time luminosity constraints with ${ }^{48} \mathrm{~V}$ decay, we would require nearly full gammaray trapping $\left(\sim 0.05\right.$ mag day ${ }^{-1}$, five times steeper than $\left.{ }^{56} \mathrm{Co}\right)$, which is inconsistent with our low derived ejecta mass. Thus, although we cannot completely rule out some (especially early) contributions from other radioactive decay chains, we find that our observations are consistent with SN 2005ek being powered by the radioactive decay of $\sim 0.03 M_{\odot}$ of ${ }^{56} \mathrm{Ni}$. We summarize this and our other inferred explosion parameters in Table 6. 
Table 6

Derived Explosion Parameters

\begin{tabular}{lcc}
\hline \hline Parameter & Unit & Value \\
\hline$L_{\text {bol,peak }}$ & $10^{42} \mathrm{erg} \mathrm{s}^{-1}$ & $1.2 \pm 0.2$ \\
$E_{\text {rad }}{ }^{2}$ & $10^{47} \mathrm{erg}$ & $8.2 \pm 0.3$ \\
$M_{\mathrm{ej}}$ & $M_{\odot}$ & $0.3-0.7$ \\
$E_{K}$ & $10^{51} \mathrm{erg}$ & $0.25-0.52$ \\
$M_{\mathrm{Ni}}$ & $M_{\odot}$ & $0.02-0.03$ \\
$v_{\text {phot, } \max }$ & $\mathrm{km} \mathrm{s}^{-1}$ & $8500 \pm 500$ \\
\hline
\end{tabular}

Note. ${ }^{\text {a }}$ Emitted between -1 and +16 days.

\section{HOST GALAXY: UGC 2526}

\subsection{Global Properties}

SN 2005ek exploded in the outskirts of UGC 2526, an edge-on spiral galaxy of morphology Sb. In Figure 15 we show the SED of UGC 2526, which was compiled from the SDSS (Ahn et al. 2012) and IRAS (Miville-Deschênes \& Lagache 2005) catalogs and supplemented with upper limits from our radio observations described in Section 2. Also shown is an Sb model template from the SWIRE database (Silva et al. 1998). Using this template, we derive a star-formation rate (SFR) of $\sim 2-5 M_{\odot} \mathrm{yr}^{-1}$ (Yun \& Carilli 2002; Kennicutt 1998). SN 2010X, SN 2002bj, and SN 1885A also exploded in star-forming galaxies.

UGC 2526 has a low radio luminosity when compared to the $\mathrm{Sb}$ template which provides a best fit at other wavelengths. According to Chakraborti et al. (2012) this can be explained if electrons responsible for producing the radio synchrotron emission undergo significant inverse-Compton losses. Synchrotron and inverse-Compton losses are proportional to the energy density in magnetic fields and seed photons, respectively. The energy density of a characteristic Milky-Way-like magnetic field of $\sim 5 \mu \mathrm{G}$ is $\sim 10^{-12} \mathrm{erg} \mathrm{cm}^{-3}$. We estimate that the bolometric luminosity of the host galaxy contributes an energy density of $\sim 3 \times 10^{-12} \mathrm{erg} \mathrm{cm}^{-3}$ which could lead to significant inverse-Compton losses.

We measure the metallicity of UGC 2526 using our hostgalaxy spectrum centered on the galaxy nucleus (Section 2). $\mathrm{H} \alpha$ and $\left[\mathrm{N}_{\mathrm{II}}\right]$ line fluxes were measured using the Markov Chain Monte Carlo technique described by Sanders et al. (2012) and, using the relations of Pettini \& Pagel (2004), we find the metallicity of the UGC 2526 to be $12+\log (\mathrm{O} / \mathrm{H})_{\mathrm{PP} 04 \mathrm{~N} 2}=$ $8.79 \pm 0.06$. This value is approximately solar. ${ }^{23}$ This metallicity measurement may be affected by absorption from the underlying stellar population, although $\mathrm{H} \alpha$ should be relatively less affected than $\mathrm{H} \beta$, resulting in a slight overestimation of the actual metallicity. For typical SN host galaxies the correction factor is on the order of $0.05 \mathrm{dex}$ and in extreme cases $\sim 0.2 \mathrm{dex}$. Additionally, the relatively high $[\mathrm{N}$ II] contribution suggests that the galaxy may be weakly active (probably a LINER; e.g., Ho et al. 1997).

\subsection{Explosion-site Properties}

The explosion site of SN 2005ek is offset nearly $30 \mathrm{kpc}(1.5)$ in projection from the center of UGC 2526 , which possesses a major diameter of $D_{25} \approx 69 \mathrm{kpc}$. This places SN 2005ek at the extreme high end of the distribution of offsets seen for all SN subtypes (Prieto et al. 2008; Kelly \& Kirshner 2012), which is especially notable because $\mathrm{SNe} \mathrm{Ib} / \mathrm{Ic}$ typically exhibit smaller

$\overline{2312+\log (\mathrm{O} / \mathrm{H})_{\text {solar }}=8.69 \text { on the PP04N2 }}$ scale.

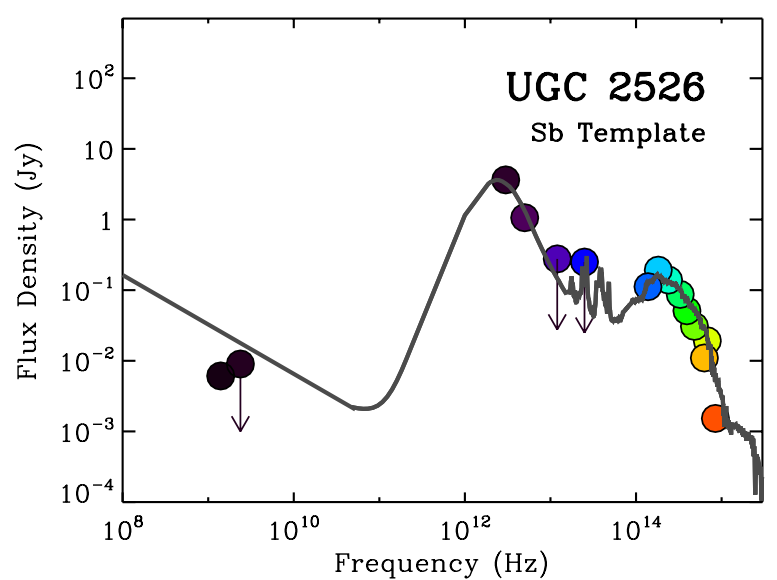

Figure 15. SED for UGC 2526 (circles) and the best-fit Sb galaxy model (gray line).

(A color version of this figure is available in the online journal.)

offsets than SNe II and SNe Ia. This large offset, coupled with the observation of metallicity gradients in many spiral galaxies (Zaritsky et al. 1994), implies that the explosion-site metallicity is likely lower than the value we measured in the galaxy nucleus. In contrast, SN 2010X, SN 2002bj, and SN 1885A all exploded with low projected offsets (Kasliwal et al. 2010; Poznanski et al. 2010; Perets et al. 2011).

The lack of nebular emission lines in the explosion-site spectrum allows us to place a strict limit on the amount of star formation within the $1^{\prime \prime}$ slit $(\sim 0.3 \mathrm{kpc}$ at the distance of UGC 2526). We measure a $3 \sigma$ upper limit on the $\mathrm{H} \alpha$ line flux of $3.3 \times 10^{37} \mathrm{erg} \mathrm{s}^{-1}$, which corresponds to an upper limit on the local SFR of $2.6 \times 10^{-4} M_{\odot} \mathrm{yr}^{-1}$ (Equation (2), Kennicutt 1998). While this value is an order of magnitude below the mean $\mathrm{H} \alpha$ flux measured for $\mathrm{H}$ II regions associated with core-collapse SNe in the sample of Crowther (2013), negligible $\mathrm{H} \alpha$ flux at the explosion site of a core-collapse SN is not unprecedented. Only 21 of $39 \mathrm{SNe}$ in the Crowther (2013) sample show evidence for an associated $\mathrm{H}$ II region.

However, a much higher fraction of $\mathrm{SNe} \mathrm{Ib} / \mathrm{Ic}$ are associated with $\mathrm{H}$ II regions compared to $\mathrm{SNe}$ II $(70 \% \pm 26 \%$ versus $38 \% \pm 11 \%$; Crowther 2013). This trend may reflect the fact that massive stars with $M \lesssim 12 M_{\odot}$ (which are expected to explode as $\mathrm{SNe}$ II) have lifetimes longer than the roughly 20 Myr lifetimes of giant $\mathrm{H}$ II regions. Thus, the lack of observed H II emission makes the explosion site of SN 2005ek more comparable to those of SN II and is consistent with a progenitor older than $\sim 20$ Myr.

\section{RATES}

SN 2005ek was discovered as part of the LOSS survey, which achieved a high level of completeness. From the LOSS data, Li et al. (2011) constructed volume-limited samples of Type Ia and core-collapse SNe out to distances of $80 \mathrm{Mpc}$ and $60 \mathrm{Mpc}$, respectively, which were used to derive relative rates for various SN subtypes. Although SN 2005ek was not included in this original analysis, ${ }^{24}$ we can use the LOSS data to obtain a rough estimate for the relative rate of SN $2005 \mathrm{ek}$-like transients. SN 2002bj was also discovered by the LOSS survey, and we derive two sets of rates below, one of which assumes SN 2002bj and SN 2005ek are members of the same class of objects.

\footnotetext{
24 Its distance of $66 \mathrm{Mpc}$ and classification as an SN Ic placed it just slightly outside the relevant sample volume (60 Mpc for core-collapse $\mathrm{SNe}$ ).
} 
The LOSS volume-limited SN Ia sample contains 74 objects and is $99 \%$ complete to a distance of $80 \mathrm{Mpc}$. In order to estimate the incompleteness correction for SN 2005ek, we examine the correction factors for SN 2002dk and SN 2002jm, two SN 1991bg-like objects with peak magnitudes similar to that of SN 2005ek. Li et al. (2011) find that both objects are $\sim 97 \%$ complete to a distance of $80 \mathrm{Mpc}$. This correction factor is based on a combination of peak magnitude and light-curve shape, and should therefore be taken as an upper limit for the completeness factor of the rapidly declining SN 2005ek. If the completeness factor of SN $2005 \mathrm{ek}$ lies between $50 \%$ and $100 \%$, we may estimate that the rate of such transients is $\sim 1 \%-2 \%$ of the SN Ia rate. If we include SN 2002bj in the same category of objects as $\mathrm{SN} 2005 \mathrm{ek}$, this rate rises to $\sim 2 \%-3 \%$ of the $\mathrm{SN}$ Ia rate. These rates should be taken as lower limits, and are consistent with those estimated by Poznanski et al. (2010) and Perets et al. (2011). It should also be noted that Poisson errors in this small number regime are large.

\section{POSSIBLE PROGENITOR CHANNELS}

The observations and analysis presented above allow us to examine several possible progenitor models for SN 2005ek. SN 2005ek shows a very rapid post-maximum decline, a peak luminosity of $\sim 10^{42} \mathrm{erg} \mathrm{s}^{-1}$, colors which redden with time, and photospheric velocities which evolve from $\sim 9000 \mathrm{~km} \mathrm{~s}^{-1}$ near maximum to $\sim 7000 \mathrm{~km} \mathrm{~s}^{-1}$ at +9 days. Spectroscopic modeling reveals a small ejecta mass $\left(0.3-0.7 M_{\odot}\right)$ which is predominantly oxygen $(\sim 85 \%)$, with smaller amounts of other intermediate-mass elements $(\mathrm{Mg}, \mathrm{C}, \mathrm{Si})$ and an explosion kinetic energy of $(2.5-5.0) \times 10^{50} \mathrm{erg}$. The pseudo-bolometric light curve is consistent with an explosion powered by $\sim 0.03 M_{\odot}$ of ${ }^{56} \mathrm{Ni}$, assuming a non-negligible fraction of the gamma-rays escape at early times. This assumption is consistent with our low inferred ejecta mass and the emergence of nebular features at only nine days post-maximum light. Finally, both the large offset and low level of $\mathrm{H} \alpha$ emission from the explosion site of SN 2005ek are consistent with a progenitor older than $~ 20 \mathrm{Myr}$.

A robust progenitor model should be able to reproduce all of the above properties. In addition, if one accepts that the spectroscopic and compositional similarities between SN 2005ek and other normal SN Ic imply that they should have a common class of progenitors, the model should be capable of producing a variety of observed decline rates and ejecta masses. Possible progenitor channels can be divided into two classes: those involving a WD or neutron star (NS) and those involving a massive star. We examine both below.

\subsection{Degenerate Objects}

Explosion models involving degenerate objects make attractive models for faint, rapidly evolving transients, as they naturally predict small ejecta masses. In addition, the old stellar environment of SN $2005 \mathrm{ek}$ is consistent with a progenitor system containing at least one degenerate object. Here we discuss several specific scenarios in the context of SN 2005ek: the accretion-induced collapse (AIC) of a WD, a WD-NS or an NS-NS merger, and the detonation of a helium shell on a low-mass WD.

\subsubsection{Accretion-induced Collapse (AIC)}

Under certain circumstances, when an accreting WD nears the Chandrasekhar mass, electron capture may occur in its core, causing it to collapse to an NS rather than undergo a thermonuclear explosion (Nomoto \& Kondo 1991). Modern simulations suggest that the subsequent bounce and neutrino-driven wind can lead to the ejection of a small amount of material, producing a weak, rapidly evolving transient powered by radioactive decay (e.g., Metzger et al. 2009; Darbha et al. 2010; Fryer 1999; Fryer et al. 2009). The observational properties of these AIC transients should vary if the AIC is caused by the merger of two WDs rather than the collapse of a single-degenerate object.

The single-degenerate case is unlikely to produce a transient similar to SN 2005ek. The simulations of Dessart et al. (2006) predict ejecta masses and explosion energies of $\sim 10^{-2} M_{\odot}$ and $\sim 10^{49}$ erg, respectively, an order of magnitude below those inferred for SN 2005ek. This is evident in the far left panel of Figure 16, where the theoretical light curves of Darbha et al. (2010; black lines) are significantly faster and fainter than those of SN 2005ek (red stars). In addition, the predicted velocities are on the order of $0.1 c$, and a majority of the ejecta is likely processed to nuclear statistical equilibrium, implying a dearth of intermediate-mass elements (Darbha et al. 2010; Metzger et al. 2009; Fryer 1999).

An AIC due to the merger of two WDs may be "enshrouded" (Metzger et al. 2009) by $\sim 0.1 M_{\odot}$ of unburned material left in a remnant disk (Yoon et al. 2007). This material will be shock heated by the ensuing explosion, potentially synthesizing intermediate-mass elements, as well as slowing the initially rapid ejecta velocity. However, models of Fryer et al. (2009) suggest that this heating may lead to a transient which peaks in the UV bands, inconsistent with our observations of SN 2005ek.

\subsection{2. $W D-N S / N S-N S$ Merger}

Both NS-NS and NS-WD mergers have also been theorized to produce faint optical transients. In the former case, $r$-process nucleosynthesis is thought to occur during the ejection of neutron-rich tidal tails, yielding a rapidly evolving transient with peak luminosities between $10^{41}$ and $10^{42} \mathrm{erg} \mathrm{s}^{-1}$ (Metzger et al. 2010; Roberts et al. 2011; solid line, middle left panel of Figure 16). However, similar to the single-degenerate AIC scenario described above, the ejecta masses are lower $\left(\sim 10^{-2} M_{\odot}\right)$, ejecta velocities are higher $(\sim 0.1 c)$ and the nucleosynthetic yields are inconsistent (mainly $r$-process elements) with our observations of SN 2005ek. In addition, using improved $r$-process opacities Barnes \& Kasen (2013) find that these transients may be fainter, longer lived, and significantly redder than previously hypothesized.

In contrast, the tidal disruption of a WD by an NS or black hole $(\mathrm{BH})$ yields a set of explosion parameters at least broadly consistent with those observed for SN 2005ek. By examining the evolution of and nucleosynthesis within the accretion disk formed during the disruption Metzger (2012) produce a set of models with ejecta masses between 0.3 and $1.0 M_{\odot}$, ejecta velocities between 1000 and $5000 \mathrm{~km} \mathrm{~s}^{-1}$, synthesized nickel masses between $10^{-3}$ and $10^{-2} M_{\odot}$, and peak luminosity between $10^{39}$ and $10^{41.5} \mathrm{erg} \mathrm{s}^{-1}$. The disruption of a larger WD yields a transient with a larger ejecta mass, expansion velocity, and nickel mass. Two example light curves are shown as dashed lines in the middle left panel of Figure 16. Although the curves fall slightly below our observations (a consequence of the slightly lower inferred nickel mass) the shape is reproduced. In addition, because the outer layers of the disk do not burn to nuclear statistical equilibrium, the final ejecta composition is at least qualitatively consistent with our results for $\mathrm{SN} 2005 \mathrm{ek}$ (mainly $\mathrm{O}, \mathrm{C}, \mathrm{Si}, \mathrm{Mg}, \mathrm{Fe}$, and $\mathrm{S})$, although the exact compositional fractions may not be reproduced. In particular, in order to synthesize enough ${ }^{56} \mathrm{Ni}$ 


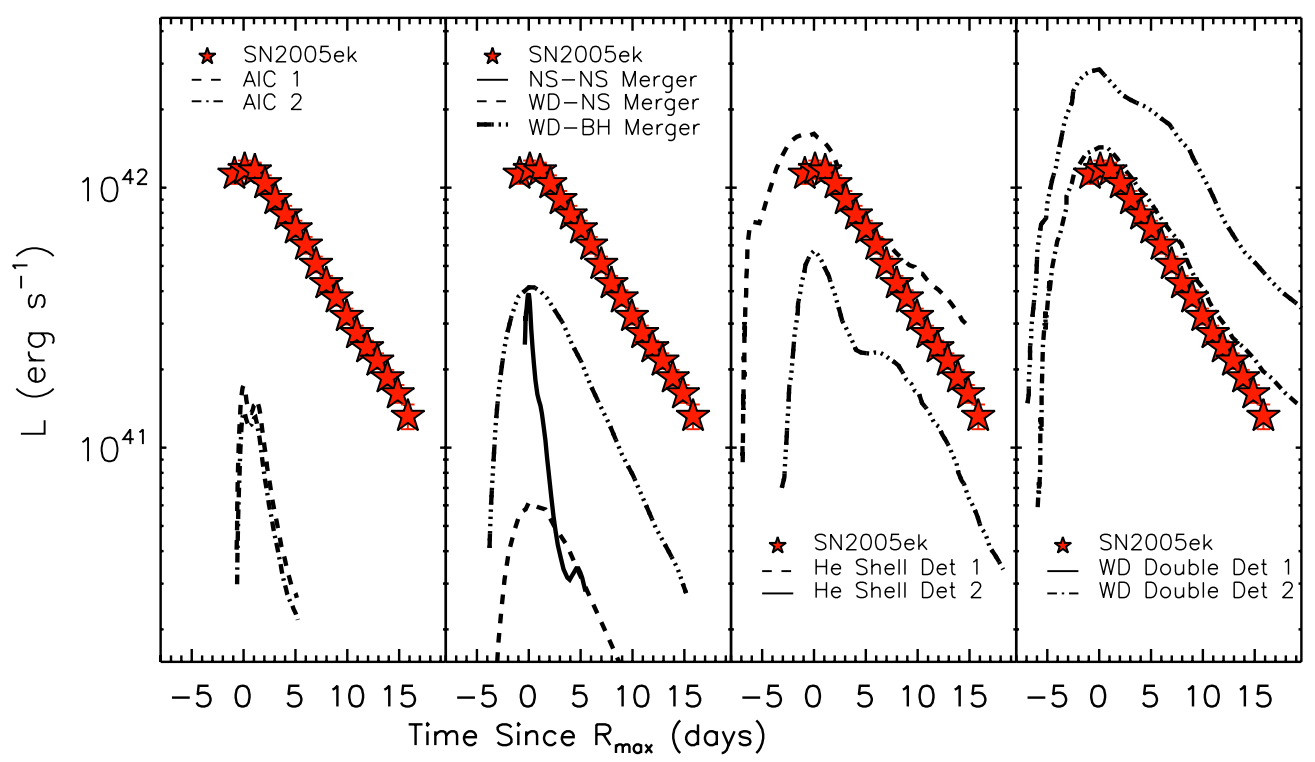

Figure 16. Comparison of the pseudo-bolometric light curve of SN 2005ek (red stars) to theoretical light curves. Far left: two AIC models from Darbha et al. (2010), Both models are calculated for $M_{\mathrm{ej}}=10^{-2} M_{\odot}$. Middle left: the NS-NS merger from Metzger et al. (2010), the WD-BH merger from Metzger (2012), and the WD-NS merger from Metzger (2012). Middle right: two ".Ia" models from (Shen et al. 2010). Models 1 and 2 represent the detonation of a $0.1 M_{\odot}$ He shell on a $1.0 M_{\odot} \mathrm{WD}$ and a $0.05 M_{\odot}$ He shell on a 1.2 $M_{\odot} \mathrm{WD}$, respectively. Far right: two edge-lit double-detonation models from Sim et al. (2012). Models 1 and 2 represent a $0.21 M_{\odot}$ He shell on a $0.58 M_{\odot}$ and $0.45 M_{\odot} \mathrm{WD}$, respectively.

(A color version of this figure is available in the online journal.)

to power SN 2005ek, the current models would require a total ejecta mass greater than $1.0 \mathrm{M}_{\odot}$.

\subsubsection{Helium-shell Detonation}

Finally, we examine the detonation of a helium shell on the surface of a WD, a model for which numerous theoretical light curves and spectra have been produced (e.g., Woosley et al. 1986; Shen et al. 2010; Fryer et al. 2009; Waldman et al. 2011; Sim et al. 2012) and which has been invoked to explain a number of unusual recent transients (e.g., SN 2005E, Perets et al. 2010; SN 2002bj, Poznanski et al. 2010; SN 2010X, Kasliwal et al. 2010; SN 1885A, Chevalier \& Plait 1988). The term “.Ia," which is often associated with this explosion mechanism, was used by Bildsten et al. (2007) to describe the specific case where the detonation occurs after mass transfer within an AM CVn binary system. In this case, the predicted helium-shell mass at the time of detonation is relatively small $\left(\lesssim 0.1 M_{\odot}\right)$, which leads to a faint $\left(-15>M_{R}>-18 \mathrm{mag}\right)$ and rapidly evolving transient. In the middle right panel of Figure 16, we show two ".Ia" model light curves from Shen et al. (2010). SN 2005ek falls comfortably between the two.

However, despite the similarity in light-curve morphology, the ".Ia" model in its basic form fails to reproduce the inferred abundances of SN 2005ek. The detonation of a predominately helium shell should yield ejecta dominated by calcium, ironpeak elements (especially titanium), and unburned helium, with a notable absence of other intermediate-mass elements (Shen et al. 2010; Perets et al. 2010). As discussed in Section 4.5, it is not straightforward to produce a high oxygen abundance from helium burning, and although calcium and titanium features are present in the spectra of both SN 2005ek and SN 2010X, these do not correspond to high abundances.

One possible reconciliation of these issues is if the heliumshell detonation triggers a second detonation within the $\mathrm{C}-\mathrm{O}$ WD. Such "double-detonation" scenarios in solar-mass WDs have been thoroughly investigated as a possible explosion mechanism for normal SNe Ia. However, Sim et al. (2012) extend this analysis to low-mass WDs, considering both core-compression and edge-lit secondary detonations. In the former case significant amounts of iron-peak elements are synthesized and a much more slowly evolving light curve is produced. However, in the latter case the main modification to the observable parameters is the production of additional intermediate-mass elements. Depending on the precise abundances synthesized and amount of unburned $\mathrm{C}-\mathrm{O}$ material ejected, it is possible that this scenario can explain most of the observational properties of SN 2005ek. In the far-right panel of Figure 16 we show two edge-lit models from Sim et al. (2012), demonstrating that they are capable of reproducing the morphology of SN $2005 \mathrm{ek}$.

\subsection{Massive Stars}

In our above discussion, we found that both the edge-lit double detonation of a low-mass WD and the tidal disruption of a WD by an NS could potentially explain the observed properties of SN 2005ek. However, as discussed in Section 4, SN 2005ek shows remarkable spectroscopic similarity to a number of normal $\mathrm{SNe}$ Ic. If one assumes a single set of progenitors for these objects, and accepts their inferred ejecta masses (between 1 and $10 M_{\odot}$; Mazzali et al. 2009, 2013), it is difficult to reconcile the data with a WD progenitor. We therefore now examine the possibility that the progenitor of SN 2005ek was a massive star.

The inferred ejecta mass and abundances provide some constraints on the pre-explosion mass and envelope density structure of any massive-star progenitor for SN 2005ek. With typical core-collapse SN producing a compact remnant having a mass $\gtrsim 1.3 M_{\odot}$ (Fryer \& Kalogera 2001), the ejecta of SN 2005ek would have only constituted a small fraction of the pre-explosion progenitor mass. At the same time, the presence of a small but non-negligible amount of $\mathrm{Ni}, \mathrm{Si}$, and $\mathrm{Mg}$ implies 
that a portion of the ejecta was nuclearly processed during the explosion. In this context, we discuss three potential massivestar explosion mechanisms for SN 2005ek: an iron-core-collapse $\mathrm{SN}$, an electron-capture SN, and a fallback SN.

\subsubsection{Iron-core-collapse $S N$}

Stars with masses $\gtrsim 11 M_{\odot}$ are expected to proceed through silicon burning before undergoing an iron-core collapse at the ends of their lives. While some amount of fallback is expected in all core-collapse SNe (Fryer 1999; Woosley \& Heger 2002), for stars with initial masses $\lesssim 20 M_{\odot}$ the final remnant mass will be dominated by the mass of the iron core at the time of collapse (i.e., approximately the Chandrasekhar mass; we will examine the case of more significant fallback in Section 8.2.3). In this case, we would infer a pre-explosion mass for SN 2005ek of $\sim 2 M_{\odot}$. This may additionally apply for stars with initial masses around $\sim 50 M_{\odot}$ in the case of strong Wolf-Rayet winds (Woosley \& Heger 2002; see their Figure 16).

The environment of SN 2005ek makes the latter situation (a high-mass progenitor with exceptionally strong Wolf-Rayet winds) less likely. With a large offset from the center of its host (see Section 6) and the metallicity gradients common in spiral galaxies (Zaritsky et al. 1994), it is likely that SN 2005ek exploded in a low-metallicity environment. In this case, mass loss from massive stars should be reduced rather than enhanced (Vink et al. 2001). In addition, such a high-mass progenitor would necessarily be short lived, and the lack of $\mathrm{H} \alpha$ flux at the explosion site is more consistent with a lower-mass progenitor $\left(\lesssim 12 M_{\odot}\right.$; Section 6$)$.

For a lower-mass progenitor, it would likely be necessary to invoke binary stripping as stars of this initial mass are not expected to strip their hydrogen envelopes via winds. This situation would be similar to that described by Nomoto et al. (1994) for SN 1994I. In that model, a star having an initial mass of $\sim 15 M_{\odot}$ was stripped via binary interaction, yielding a $\mathrm{C}-\mathrm{O}$ star of $\sim 2 M_{\odot}$ which underwent core collapse. Recall that the spectra of SN 1994I closely resembled those of SN 2005ek (Figure 9) and its decline rate was intermediate between SN 2005ek and the bulk of other SNe Ib/Ic (Figure 5). The ejecta mass (Nomoto et al. 1994) derived for SN 1994I was $0.88 M_{\odot}$, slightly larger than that inferred for SN $2005 \mathrm{ek}$. Taken at face value, it would be necessary to invoke either a star having a smaller initial mass (only slightly above the lower limit for iron-core collapse, consistent with the host environment described above) or more extreme stripping to reproduce $\mathrm{SN} 2005 \mathrm{ek}$.

In either case, we would expect the amount of radioactive material produced to be on the low side for $\mathrm{SNe} \mathrm{Ib} / \mathrm{Ic}$ (due to the small amount of material at sufficient densities) and the composition to be dominated by oxygen, along with carbon and neon. Both predictions are well matched by our observations of SN 2005ek. The ejecta abundances we derive for SN 2005ek are very similar to those found for SN 2007gr (Mazzali et al. 2010) and SN 1994I (Sauer et al. 2006). If SN 2005ek is due to the iron-core collapse of a massive star, it exhibits one of the lowest kinetic energies $\left(2.5-5.2 \times 10^{50} \mathrm{erg}\right)$ and most extreme ratios of ejecta mass to remnant mass $\left(M_{\mathrm{ej}} / M_{\text {remnant }}<1.0\right)$ ever observed. However, the inferred ratio of kinetic energy to ejecta mass is similar to that of other SNe Ic $\left(E_{K, 51} / M_{\mathrm{ej}, \odot} \approx 1\right)$.

Detailed modeling would be necessary to determine if the low kinetic energy inferred for SN 2005ek is consistent with what one would expect from the iron-core collapse of a stripped lowmass progenitor. Fryer \& Kalogera (2001) argue that although stripping the hydrogen envelope from a massive star should not significantly impact the resulting explosion energy, the same may not be true if the stripping extends into the $\mathrm{C}-\mathrm{O}$ core (as is the case for $\mathrm{SNe}$ Ic). In deriving the distribution of remnant masses, Fryer \& Kalogera (2001) assume an explosion energy which is proportional to the $\mathrm{C}-\mathrm{O}$ core mass, an assumption which is qualitatively consistent with the low kinetic energy inferred for SN 2005ek.

\subsubsection{Electron-capture $S N$}

If the progenitor of SN 2005ek was a relatively low-mass star, it is possible that the ensuing explosion was due to a (stripped) electron-capture $\mathrm{SN}$ rather than a traditional iron-core-collapse event. Stars within a narrow mass range $\left(\sim 8-10 M_{\odot}\right.$; Nomoto 1987; Woosley \& Weaver 1980; Iben et al. 1997; Kitaura et al. 2006) are expected to undergo electron capture in their $\mathrm{O}-\mathrm{Ne}-\mathrm{Mg}$ core, decreasing pressure support, and causing the core to collapse to an NS (Nomoto 1987). Kitaura et al. (2006) have shown that the explosion proceeds in a similar manner to that of an iron-core-collapse SN (e.g., a delayed explosion driven by neutrinos), although a lower-energy explosion is produced and the density structure of the overlying material differs. The explosion energy found in the models of Kitaura et al. (2006) is $\sim 10^{50} \mathrm{erg}$, comparable to the kinetic energy inferred for SN 2005ek. However, the envelopes surrounding $\mathrm{O}-\mathrm{Ne}-\mathrm{Mg}$ cores are expected to be relatively diffuse. This leads to an explosion which synthesizes only very small amounts of ${ }^{56} \mathrm{Ni}\left(\sim 10^{-3}\right.$; Kitaura et al. 2006; Bethe \& Wilson 1985) and ejecta which show few signs of nuclear processing. On both these points, our observations of SN 2005ek are more consistent with a low-mass iron-core-collapse event than with an electron-capture SN.

\subsubsection{Fallback $S N$}

Alternatively, it is possible that the low ejecta mass of SN 2005ek is not caused by a low pre-explosion mass, but by a significant amount of fallback onto the proto-NS. Qualitatively, this is expected to occur when the binding energy of the outer envelope is high, although the actual fallback criteria are complex, depending on the evolution of the shock velocity within the envelope as well as parameters such as metallicity and rotation. It has been theorized that significant fallback should occur for stars with masses $\gtrsim 30 M_{\odot}$ (Fryer \& Kalogera 2001; MacFadyen et al. 2001). Although the successful ejecta in such explosions can possess low kinetic energies (MacFadyen et al. 2001), events of this sort lacking a hydrogen envelope are expected to be quite faint, as a majority of the radioactive ${ }^{56} \mathrm{Ni}$ is synthesized in the inner portions of the ejecta (which fall back onto the proto-NS). The peak luminosity of SN 2005ek would likely require a non-negligible amount of mixing prior to fallback. Additionally, the environment-based arguments against a very massive progenitor for SN 2005ek (Section 8.2.1) still hold.

\subsection{Applicability of Conclusions to Other Rapidly Declining Events}

In the discussion above we found that SN 2005ek could potentially be explained by either the edge-lit double detonation of a low-mass WD, the tidal disruption of a WD by an NS, or the iron-core collapse of stripped massive star, with the latter option preferred if one takes the strong spectroscopic similarity of SN 2005ek to normal SNe Ic to be an indication of a similar 
progenitor channel. However, we note that these conclusions may not broadly apply to all of the rapidly declining SNe I in the literature to date. While SN2005ek, SN 2010X, SN 2002bj, SN 1885A, and SN 1939B all exhibit similar post-maximum decline rates and have low inferred ejecta masses $\left(\lesssim 0.3 M_{\odot}\right)$, only SN 2010X possess a similar peak luminosity and spectroscopic evolution to SN 2005ek. SN 2002bj, SN 1939B, and SN 1885 are all significantly ( $\gtrsim 1.3 \mathrm{mag}$ ) more luminous. Coupled with their fast decline rate it is unclear whether a similar modeling scheme evoked in Section 5 would produce a self-consistent solution for these events. SN 2002bj also shows significantly bluer colors, slower expansion velocities, and a distinct spectroscopic evolution near maximum. In addition, while we argue that the explosion site of SN 2005ek does not rule out the possibility of a massive-star progenitor (by analogy with explosion sites of many SN IIP), SN 1939B exploded in an elliptical galaxy and SN 1885A (which exploded in the bulge of M31) shows no signs of an NS in its remnant (see, Perets et al. 2011). In short, while the conclusions above likely also apply to the rapidly evolving SN 2010X, their validity with respect to SN 2002bj, SN 1885A, and SN 1939B is less clear. More detailed modeling will be required to distinguish various possibilities. Such modeling is currently underway for SN 2010X (K. W. Kleiser et al., in preparation).

\section{SUMMARY AND CONCLUSIONS}

We have presented the discovery and extensive multiwavelength observations of the rapidly evolving Type I SN 2005ek. Here we summarize our main conclusions.

1. Reaching a peak of $M_{R}=-17.3 \mathrm{mag}$ and declining by $\sim 3 \mathrm{mag}$ in the first 15 days post-maximum, SN 2005ek is one of the fastest declining SNe I known thus far.

2. Late-time photometric detections show a shallower decay timescale which is similar to the late-time evolution of other SNe I.

3. The spectra of SN 2005ek closely resemble those of other SNe Ic in both morphology and velocity. However, SN 2005ek enters the optically thin phase at a much earlier epoch. We present evidence for the onset of nebular spectroscopic features at only nine days post-maximum.

4. The bolometric light curve of SN 2005ek peaks at $\sim 10^{41} \mathrm{erg} \mathrm{s}^{-1}$. Its evolution is consistent with an explosion powered by $\sim 0.03 M_{\odot}$ of ${ }^{56} \mathrm{Ni}$, with incomplete gamma-ray trapping at early times.

5. We estimate the ejecta mass and kinetic energy of SN 2005ek to be $0.3-0.7 M_{\odot}$ and $2.5-5.2 \times 10^{50} \mathrm{erg}$, respectively.

6. SN 2005ek exploded in a star-forming galaxy, but with a large projected offset in an area lacking strong $\mathrm{H} \alpha$ emission.

7. The ejecta of SN $2005 \mathrm{ek}$ are dominated by oxygen $(\sim 86 \%)$. Other intermediate-mass elements $(\mathrm{C}, \mathrm{Mg}, \mathrm{Si}, \mathrm{S}, \mathrm{Ca})$ account for $\sim 13.5 \%$ of the ejecta, while iron-peak elements make up only $0.5 \%$. These oxygen-dominated ejecta are inconsistent with the helium-shell detonation model (".Ia") which has previously been invoked to explain such rapidly declining events.

8. Many of the observed properties of SN 2005ek could be explained by either the edge-lit double detonation of a low-mass WD or the tidal disruption of a WD by an NS. However, if we assume that the strong spectroscopic similarities between SN 2005ek and other normal SNe Ic (with a wide range of decline timescales and inferred ejecta masses) to be an indication of a similar progenitor channel, a WD progenitor becomes very unlikely.

9. Our observations and modeling of SN 2005ek are also consistent with the iron-core collapse of a low-mass star $\left(12-15 M_{\odot}\right)$, stripped by binary interaction. In particular, the abundances derived are very similar to those found for other SN Ic. In this case, SN $2005 \mathrm{ek}$ may possess the most extreme ratio of ejecta mass to remnant mass observed for a core-collapse $\mathrm{SN}$ to date. The ratio of kinetic energy to ejecta mass is similar to that of other SNe Ic.

10. The rate of such rapidly declining $\mathrm{SNe} I$ is at least $1 \%-3 \%$ of the normal SN Ia rate.

11. Based on their strong photometric and spectroscopic similarities, our conclusions likely also apply to the rapidly evolving Type I SN 2010X. However, despite their similar decline rates, there are several important observational differences between SN 2005ek and SN 2002bj, SN 1939B, and SN 1885A. More detailed analysis will be required to determine if these three (more luminous) objects belong to the same class of explosions.

We thank L. Bildsten, K. Shen, and T. Janka for helpful discussions. We are grateful to the staffs at the numerous observatories where we gathered data. We kindly acknowledge Sung Park, Katsuki Shimasaki, and Tom Matheson for assisting in the acquisition of some of the observations presented here. We acknowledge useful conversations at a Sky House workshop.

M.R.D. is supported in part by the NSF through a Graduate Research Fellowship. Support for this work was provided by the David and Lucile Packard Foundation Fellowship for Science and Engineering awarded to A.M.S. J.M.S. is supported by an NSF Astronomy and Astrophysics Postdoctoral Fellowship under award AST-1302771. A.S.F. acknowledges support from the U.S. National Science Foundation (NSF) under grant SES 1056580.

This work was supported in part by the National Science Foundation under grant No. PHYS-1066293 and the hospitality of the Aspen Center for Physics. We also acknowledge the hospitality of the Kavli Institute for Theoretical Physics and partial support by the National Science Foundation under grant No. NSF PHY11-25915.

The W. M. Keck Observatory, which is operated as a scientific partnership among the California Institute of Technology, the University of California, and NASA; the observatory was made possible by the generous financial support of the W. M. Keck Foundation. Observations reported here were obtained at the MMT Observatory, a joint facility of the Smithsonian Institution and the University of Arizona. The Hobby-Eberly Telescope (HET), is a joint project of the University of Texas at Austin, the Pennsylvania State University, Stanford University, Ludwig-Maximilians-Universität München, and Georg-AugustUniversität Göttingen. The HET is named in honor of its principal benefactors, William P. Hobby and Robert E. Eberly. The Very Large Array is a facility of the National Science Foundation operated under cooperative agreement by Associated Universities, Inc. PAIRITEL is operated by the Smithsonian Astrophysical Observatory (SAO) and was made possible by a grant from the Harvard University Milton Fund, the camera loan from the University of Virginia, and the continued support of the SAO and UC Berkeley. We thank M. Skrutskie for his continued support of the PAIRITEL project.

The supernova research of A.V.F.'s group at UC Berkeley is supported by Gary \& Cynthia Bengier, the Richard \& 
Rhoda Goldman Fund, the Christopher R. Redlich Fund, the TABASGO Foundation, and NSF grant AST-1211916. KAIT and its ongoing work were made possible by donations from Sun Microsystems, Inc., the Hewlett-Packard Company, AutoScope Corporation, Lick Observatory, the NSF, the University of California, the Sylvia \& Jim Katzman Foundation, and the TABASGO Foundation.

\section{REFERENCES}

Ahn, C. P., Alexandroff, R., Allende Prieto, C., et al. 2012, ApJS, 203, 21 Arnett, W. D. 1982, ApJ, 253, 785

Barnes, J., \& Kasen, D. 2013, arXiv:1303.5787

Bethe, H. A., \& Wilson, J. R. 1985, ApJ, 295, 14

Bildsten, L., Shen, K. J., Weinberg, N. N., \& Nelemans, G. 2007, ApJL, 662, L95

Blondin, S., Matheson, T., Kirshner, R. P., et al. 2012, AJ, 143, 126

Bongard, S., Baron, E., Smadja, G., Branch, D., \& Hauschildt, P. H. 2008, ApJ, 687, 456

Branch, D., Benetti, S., Kasen, D., et al. 2002, ApJ, 566, 1005

Breeveld, A. A., Landsman, W., Holland, S. T., et al. 2011, in AIP Conf. Ser. 1358, Gamma Ray Bursts 2010, ed. J. E. McEnery, J. L. Racusin, \& N. Gehrels (Melville, NY: AIP), 373

Brown, P. J., Holland, S. T., Immler, S., et al. 2009, AJ, 137, 4517

Burrows, D. N., Hill, J. E., Nousek, J. A., et al. 2005, SSRv, 120, 165

Cappellaro, E., Mazzali, P. A., Benetti, S., et al. 1997, A\&A, 328, 203

Cenko, S. B., Fox, D. B., Moon, D.-S., et al. 2006, PASP, 118, 1396

Chakraborti, S., Yadav, N., Cardamone, C., \& Ray, A. 2012, ApJL, 746, L6

Chevalier, R. A., \& Plait, P. C. 1988, ApJL, 331, L109

Clocchiatti, A., \& Wheeler, J. C. 1997, ApJ, 491, 375

Crowther, P. A. 2013, MNRAS, 428, 1927

Darbha, S., Metzger, B. D., Quataert, E., et al. 2010, MNRAS, 409, 846

Dessart, L., Burrows, A., Ott, C. D., et al. 2006, ApJ, 644, 1063

Dessart, L., Hillier, D. J., Li, C., \& Woosley, S. 2012, MNRAS, 424, 2139

de Vaucouleurs, G., \& Corwin, H. G., Jr. 1985, ApJ, 295, 287

Drout, M. R., Soderberg, A. M., Gal-Yam, A., et al. 2011, ApJ, 741, 97

Faber, S. M., Phillips, A. C., Kibrick, R. I., et al. 2003, Proc. SPIE, 4841, 1657

Fabricant, D., Cheimets, P., Caldwell, N., \& Geary, J. 1998, PASP, 110, 79

Filippenko, A. V. 1997, ARA\&A, 35, 309

Filippenko, A. V., Li, W. D., Treffers, R. R., \& Modjaz, M. 2001, in ASP Conf. Ser. 246, IAU Colloq. 183: Small Telescope Astronomy on Global Scales, ed. B. Paczynski, W.-P. Chen, \& C. Lemme (San Francisco, CA: ASP), 121

Filippenko, A. V., Richmond, M. W., Branch, D., et al. 1992, AJ, 104, 1543

Folatelli, G., Phillips, M. M., Morrell, N., et al. 2012, ApJ, 745, 74

Foley, R. J., Brown, P. J., Rest, A., et al. 2010, ApJL, 708, L61

Foley, R. J., Challis, P. J., Chornock, R., et al. 2013, ApJ, 767, 57

Foley, R. J., Chornock, R., Filippenko, A. V., et al. 2009, AJ, 138, 376

Fryer, C. 1999, ApJ, 522, 413

Fryer, C. L., Brown, P. J., Bufano, F., et al. 2009, ApJ, 707, 193

Fryer, C. L., \& Kalogera, V. 2001, ApJ, 554, 548

Gehrels, N., Chincarini, G., Giommi, P., et al. 2004, ApJ, 611, 1005

Ho, L. C., Filippenko, A. V., \& Sargent, W. L. W. 1997, ApJS, 112, 315

Howell, D. A., Sullivan, M., Nugent, P. E., et al. 2006, Natur, 443, 308

Iben, I., Jr., Ritossa, C., \& Garcia-Berro, E. 1997, ApJ, 489, 772

Kalberla, P. M. W., Burton, W. B., Hartmann, D., et al. 2005, A\&A, 440, 775

Kasliwal, M. M., Kulkarni, S. R., Gal-Yam, A., et al. 2010, ApJL, 723, L98

Kasliwal, M. M., Kulkarni, S. R., Gal-Yam, A., et al. 2012, ApJ, 755, 161

Kelly, P. L., \& Kirshner, R. P. 2012, ApJ, 759, 107

Kennicutt, R. C. 1998, ARA\&A, 36, 189

Khandrika, H., \& Li, W. 2005, CBET, 232, 1

Kitaura, F. S., Janka, H.-T., \& Hillebrandt, W. 2006, A\&A, 450, 345

Leibundgut, B., Kirshner, R. P., Phillips, M. M., et al. 1993, AJ, 105, 301

Leibundgut, B., Tammann, G. A., Cadonau, R., \& Cerrito, D. 1991, A\&AS, 89,537

Li, W., Filippenko, A. V., Chornock, R., \& Jha, S. 2003, PASP, 115, 844

Li, W., Leaman, J., Chornock, R., et al. 2011, MNRAS, 412, 1441

Lucy, L. 1999, A\&A, 345, 211

MacFadyen, A., Woosley, S., \& Heger, A. 2001, ApJ, 20, 410

Matheson, T., Filippenko, A. V., Chornock, R., Leonard, D. C., \& Li, W. 2000, AJ, 119, 2303

Matheson, T., Kirshner, R. P., Challis, P., et al. 2008, AJ, 135, 1598

Mazzali, P., \& Lucy, L. 1993, A\&A, 279, 447

Mazzali, P. A. 2000, A\&A, 363, 705

Mazzali, P. A., Deng, J., Hamuy, M., \& Nomoto, K. 2009, ApJ, 703, 1624

Mazzali, P. A., \& Lucy, L. B. 1998, MNRAS, 295, 428
Mazzali, P. A., Maurer, I., Valenti, S., Kotak, R., \& Hunter, D. 2010, MNRAS, 408,87

Mazzali, P. A., Sauer, D. N., Pastorello, A., Benetti, S., \& Hillebrandt, W. 2008, MNRAS, 386, 1897

Mazzali, P. A., Walker, E. S., Pian, E., et al. 2013, MNRAS, 432, 2463

Metzger, B. D. 2012, MNRAS, 419, 827

Metzger, B. D., Martínez-Pinedo, G., Darbha, S., et al. 2010, MNRAS, 406, 2650

Metzger, B. D., Piro, A. L., \& Quataert, E. 2009, MNRAS, 396, 1659

Miller, J. S., \& Stone, R. P. S. 1993, Lick Observatory Technical Reports, Vol. 66 (Santa Cruz, CA: Lick Observatory)

Miville-Deschênes, M.-A., \& Lagache, G. 2005, ApJS, 157, 302

Modjaz, M. 2007, PhD thesis, Harvard Univ.

Modjaz, M., Li, W., Filippenko, A. V., et al. 2001, PASP, 113, 308

Moriya, T., Tominaga, N., Tanaka, M., et al. 2010, ApJ, 719, 1445

Mould, J. R., Huchra, J. P., Freedman, W. L., et al. 2000, ApJ, 529, 786

Nomoto, K. 1987, ApJ, 322, 206

Nomoto, K., \& Kondo, Y. 1991, ApJL, 367, L19

Nomoto, K., Thielemann, F., \& Yokoi, K. 1984, ApJ, 286, 644

Nomoto, K., Yamaoka, H., Pols, O. R., et al. 1994, Natur, 371, 227

Ofek, E. O., Rabinak, I., Neill, J. D., et al. 2010, ApJ, 724, 1396

Parrent, J. T., Thomas, R. C., Fesen, R. A., et al. 2011, ApJ, 732, 30

Perets, H. B., Badenes, C., Arcavi, I., Simon, J. D., \& Gal-yam, A. 2011, ApJ, 730,89

Perets, H. B., Gal-Yam, A., Mazzali, P. A., et al. 2010, Natur, 465, 322

Pettini, M., \& Pagel, B. E. J. 2004, MNRAS, 348, L59

Phillips, M. M. 1993, ApJL, 413, L105

Phillips, M. M., Lira, P., Suntzeff, N. B., et al. 1999, AJ, 118, 1766

Pian, E., Amati, L., Antonelli, L. A., et al. 2000, ApJ, 536, 778

Poole, T. S., Breeveld, A. A., Page, M. J., et al. 2008, MNRAS, 383, 627

Poznanski, D., Chornock, R., Nugent, P. E., et al. 2010, Sci, 327, 58

Poznanski, D., Ganeshalingam, M., Silverman, J. M., \& Filippenko, A. V. 2011, MNRAS, 415, L81

Poznanski, D., Prochaska, J. X., \& Bloom, J. S. 2012, MNRAS, 426, 1465

Prieto, J. L., Stanek, K. Z., \& Beacom, J. F. 2008, ApJ, 673, 999

Richmond, M. W., van Dyk, S. D., Ho, W., et al. 1996, AJ, 111, 327

Roberts, L. F., Kasen, D., Lee, W. H., \& Ramirez-Ruiz, E. 2011, ApJL, 736, L21

Roming, P. W. A., Kennedy, T. E., Mason, K. O., et al. 2005, SSRv, 120, 95

Sanders, N. E., Soderberg, A. M., Levesque, E. M., et al. 2012, ApJ, 758, 132

Sauer, D. N., Mazzali, P. A., Deng, J., et al. 2006, MNRAS, 369, 1939

Schlegel, D. J., Finkbeiner, D. P., \& Davis, M. 1998, ApJ, 500, 525

Schmidt, G. D., Weymann, R. J., \& Foltz, C. B. 1989, PASP, 101, 713

Shen, K. J., Kasen, D., Weinberg, N. N., Bildsten, L., \& Scannapieco, E. 2010, ApJ, 715, 767

Silva, L., Granato, G. L., Bressan, A., \& Danese, L. 1998, ApJ, 509, 103

Silverman, J. M., \& Filippenko, A. V. 2012, MNRAS, 425, 1917

Silverman, J. M., Foley, R. J., Filippenko, A. V., et al. 2012, MNRAS, 425, 1789

Sim, S. A., Fink, M., Kromer, M., et al. 2012, MNRAS, 420, 3003

Smith, J. A., Tucker, D. L., Kent, S., et al. 2002, AJ, 123, 2121

Soderberg, A. M. 2007, in AIP Conf. Ser. 937, Supernova 1987A: 20 Years After: Supernovae and Gamma-Ray Bursters, ed. S. Immler, K. Weiler, \& R. McCray (Melville, NY: AIP), 492

Soderberg, A. M., Brunthaler, A., Nakar, E., Chevalier, R. A., \& Bietenholz, M. F. 2010, ApJ, 725, 922

Sutherland, P. G., \& Wheeler, J. C. 1984, ApJ, 280, 282

Taubenberger, S., Hachinger, S., Pignata, G., et al. 2008, MNRAS, 385, 75

Taubenberger, S., Pastorello, A., Mazzali, P. A., et al. 2006, MNRAS, 371, 1459

Thomas, R. C., Aldering, G., Antilogus, P., et al. 2007, ApJL, 654, L53

Thomas, R. C., Nugent, P. E., \& Meza, J. C. 2011, PASP, 123, 237

Turatto, M., Benetti, S., Cappellaro, E., Astronomico, O., \& Osservatorio, V. 2003, in From Twilight to Highlight: The Physics of Supernovae, ed. W. Hillebrandt \& B. Leibundgut (Berlin: Springer), 200

Valenti, S., Benetti, S., Cappellaro, E., et al. 2007, MNRAS, 383, 1485

Valenti, S., Elias-Rosa, N., Taubenberger, S., et al. 2008, ApJL, 673, L155

Valenti, S., Pastorello, A., Cappellaro, E., et al. 2009, Natur, 459, 674

Valenti, S., Yuan, F., Taubenberger, S., et al. 2013, arXiv:1302.2983

Vink, J. S., de Koter, A., \& Lamers, H. J. G. L. M. 2001, A\&A, 369, 574

Waldman, R., Sauer, D., Livne, E., et al. 2011, ApJ, 738, 21

Wheeler, J. C., Harkness, R. P., Clocchiatti, A., et al. 1994, ApJL, 436, L135

Wong, D. S., Park, S., Shimasaki, K., \& Filippenko, A. V. 2005, CBET, 235, 1 Woosley, S. E., \& Heger, A. 2002, RvMP, 74, 1015

Woosley, S. E., Taam, R. E., \& Weaver, T. A. 1986, ApJ, 301, 601

Woosley, S. E., \& Weaver, T. A. 1980, ApJ, 238, 1017

Yoon, S.-C., Podsiadlowski, P., \& Rosswog, S. 2007, MNRAS, 380, 933

Yun, M. S., \& Carilli, C. L. 2002, ApJ, 568, 88

Zaritsky, D., Kennicutt, R. C., Jr., \& Huchra, J. P. 1994, ApJ, 420, 87 